\title{
Multiloop amplitudes of light-cone gauge superstring field theory: odd spin structure contributions
}

\author{
Nobuyuki Ishibashi ${ }^{a}$ and Koichi Murakami ${ }^{b}$ \\ a Tomonaga Center for the History of the Universe, University of Tsukuba, \\ Tsukuba, Ibaraki 305-8571, Japan \\ ${ }^{b}$ National Institute of Technology, Kushiro College, \\ Otanoshike-Nishi 2-32-1, Kushiro, Hokkaido 084-0916, Japan \\ E-mail: ishibash@het.ph.tsukuba.ac.jp, koichi@kushiro-ct.ac.jp
}

ABSTRaCt: We study the odd spin structure contributions to the multiloop amplitudes of light-cone gauge superstring field theory. We show that they coincide with the amplitudes in the conformal gauge with two of the vertex operators chosen to be in the pictures different from the standard choice, namely $(-1,-1)$ picture in the type II case and -1 picture in the heterotic case. We also show that the contact term divergences can be regularized in the same way as in the amplitudes for the even structures and we get the amplitudes which coincide with those obtained from the first-quantized approach.

KEYwords: String Field Theory, BRST Quantization, Conformal Field Models in String Theory, Superstrings and Heterotic Strings

ARXIV EPRINT: 1712.09049 


\section{Contents}

1 Introduction 1

2 Light-cone gauge superstring field theory 2

2.1 Light-cone gauge superstring field theory 2

$\begin{array}{lll}2.2 & \text { Dimensional regularization } & 7\end{array}$

$\begin{array}{ll}2.3 & \text { The problems with odd spin structure }\end{array}$

3 Odd spin structure $\quad 10$

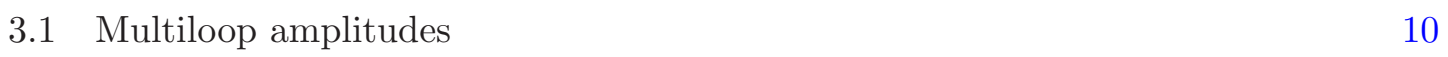

$\begin{array}{lll}3.2 & \text { Dimensional regularization } & 13\end{array}$

4 Conclusions and discussions $\quad 15$

$\begin{array}{lr}\text { A Operator valued coordinate } & 16\end{array}$

$\begin{array}{lr}\text { B Correlation functions of free fermions } & 18\end{array}$

C Dimensional regularization for odd spin structure $\quad 20$

$\begin{array}{lll}\text { C.1 Supersymmetric } X^{ \pm} \text {CFT } & 20\end{array}$

C.2 A proof of equality of (3.14) and (3.15) 24

\section{Introduction}

String field theory is expected to provide a nonperturbative formulation of string theory. It is a second-quantized string theory from which one can calculate Feynman amplitudes which agree with those of the first-quantized theory. For bosonic strings, there are several proposals of such string field theories. For superstrings, because of the problems with the method to calculate multiloop amplitudes using the picture changing operators, the construction of a string field theory has been a difficult problem. Recently, Sen has constructed a gauge invariant formulation of the string field theory for closed superstrings [1-5], based on the formulation [6] of closed string field theory for bosonic strings with a nonpolynomial action.

Light-cone gauge closed superstring field theory is a string field theory for superstrings which involves only three-string interaction terms. It can be proved formally that the Feynman amplitudes of the string field theory coincide with those of the first-quantized theory [7]. The proof was formal because there appear unphysical divergences which are called the contact term divergences [8-12]. In a previous paper [13], we have shown that these divergences can be dealt with by dimensional regularization. In the case of type II superstrings, for example, one formulates a light-cone gauge superstring field theory 
in noncritical dimensions or the one whose worldsheet theory for transverse variables is a superconformal field theory with central charge $c \neq 12$ [14]. Although Lorentz invariance is broken by doing so, it does not cause so much trouble because the light-cone gauge theory is a completely gauge-fixed theory. In [13], we have shown that the multiloop amplitudes corresponding to the Riemann surfaces with even spin structure involving external lines in the (NS,NS) sector can be calculated using the dimensional regularization and the results coincide with those of the first-quantized approach.

What we would like to do in this paper is to generalize these results to the case of the surfaces with odd spin structure. On the Riemann surfaces with odd spin structure, there exist zero modes of the fermionic variables on the worldsheet which make the manipulations of the amplitudes complicated. We will show that it is possible to deal with these zero modes and prove that the amplitudes are equal to those of the first-quantized method, when all the external lines are in the (NS,NS) sector, in the case of type II superstrings. It is straightforward to obtain similar results for heterotic strings.

The organization of this paper is as follows. In section 2, we review the results in [13] and the problems with the odd spin structures. In section 3 , we deal with the amplitudes for the odd spin structure and show that these also coincide with those from the firstquantized approach. Section 4 is devoted to discussions. In the appendices, we present details of the manipulations given in the main text.

\section{Light-cone gauge superstring field theory}

In this section, we review the known results for the multiloop amplitudes of light-cone gauge superstring field theory and the problems with the odd spin structures.

\subsection{Light-cone gauge superstring field theory}

In the light-cone gauge string field theory, the string field

$$
|\Phi(t, \alpha)\rangle
$$

is taken to be an element of the Hilbert space $\mathcal{H}$ of the transverse variables on the worldsheet and a function of

$$
\begin{aligned}
t & =x^{+}, \\
\alpha & =2 p^{+}
\end{aligned}
$$

In this paper, we consider the string field theory for type II superstrings in 10 dimensional flat spacetime as an example. $|\Phi(t, \alpha)\rangle$ should be GSO even and satisfy the level-matching condition

$$
\left(L_{0}-\bar{L}_{0}\right)|\Phi(t, \alpha)\rangle=0,
$$

where $L_{0}, \bar{L}_{0}$ are the zero modes of the Virasoro generators of the worldsheet theory. 


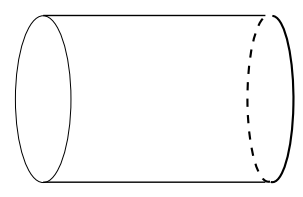

propagator

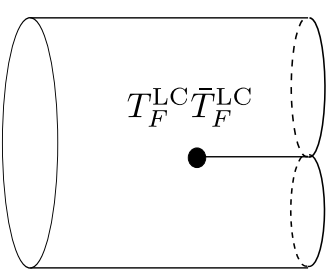

vertex

Figure 1. The propagator and the vertex of the string field theory.

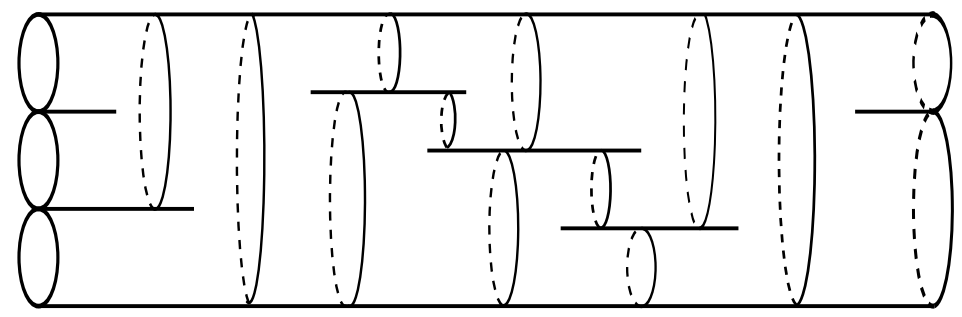

Figure 2. A Feynman diagram of strings.

The action of the string field theory is given by

$$
\begin{aligned}
& S=\int d t\left[\frac{1}{2} \sum_{\mathrm{B}} \int_{-\infty}^{\infty} \frac{\alpha d \alpha}{4 \pi}\left\langle\Phi_{\mathrm{B}}(-\alpha)\left|\left(i \partial_{t}-\frac{L_{0}+\bar{L}_{0}-1}{\alpha}\right)\right| \Phi_{\mathrm{B}}(\alpha)\right\rangle\right. \\
& +\frac{1}{2} \sum_{\mathrm{F}} \int_{-\infty}^{\infty} \frac{d \alpha}{4 \pi}\left\langle\Phi_{\mathrm{F}}(-\alpha)\left|\left(i \partial_{t}-\frac{L_{0}+\bar{L}_{0}-1}{\alpha}\right)\right| \Phi_{\mathrm{F}}(\alpha)\right\rangle \\
& -\frac{g_{s}}{6} \sum_{\mathrm{B}_{1}, \mathrm{~B}_{2}, \mathrm{~B}_{3}} \int \prod_{r=1}^{3}\left(\frac{\alpha_{r} d \alpha_{r}}{4 \pi}\right) \delta\left(\sum_{r=1}^{3} \alpha_{r}\right)\left\langle V_{3} \mid \Phi_{\mathrm{B}_{1}}\left(\alpha_{1}\right)\right\rangle\left|\Phi_{\mathrm{B}_{2}}\left(\alpha_{2}\right)\right\rangle\left|\Phi_{\mathrm{B}_{3}}\left(\alpha_{3}\right)\right\rangle \\
& \left.-\frac{g_{s}}{2} \sum_{\mathrm{B}_{1}, \mathrm{~F}_{2}, \mathrm{~F}_{3}} \int \prod_{r=1}^{3}\left(\frac{\alpha_{r} d \alpha_{r}}{4 \pi}\right) \delta\left(\sum_{r=1}^{3} \alpha_{r}\right)\left\langle V_{3} \mid \Phi_{\mathrm{B}_{1}}\left(\alpha_{1}\right)\right\rangle \alpha_{2}^{-\frac{1}{2}}\left|\Phi_{\mathrm{F}_{2}}\left(\alpha_{2}\right)\right\rangle \alpha_{3}^{-\frac{1}{2}}\left|\Phi_{\mathrm{F}_{3}}\left(\alpha_{3}\right)\right\rangle\right]
\end{aligned}
$$

which consists of the kinetic terms and the three-string interaction terms. $\sum_{\mathrm{B}}$ and $\sum_{\mathrm{F}}$ denote the sums over bosonic and fermionic string fields respectively. The three-string vertices $\left\langle V_{3}\right|$ are elements of $\mathcal{H}^{*} \otimes \mathcal{H}^{*} \otimes \mathcal{H}^{*}$ whose definition can be found in $[13,15,16]$. Here $\mathcal{H}^{*}$ denotes the dual space of $\mathcal{H}$.

It is straightforward to calculate the amplitudes by the old-fashioned perturbation theory starting from the action (2.4) and Wick rotate to Euclidean time. The propagator and the vertex are given by the worldsheets depicted in figure 1, where the left and right supercurrents $T_{\mathrm{F}}^{\mathrm{LC}}, \bar{T}_{\mathrm{F}}^{\mathrm{LC}}$ of the transverse variables $X^{i}, \psi^{i}, \bar{\psi}^{i}(i=1, \ldots, 8)$ are inserted at the interaction points of the three-string vertices. Each term in the expansion corresponds to a light-cone gauge Feynman diagram for strings.

A typical light-cone gauge Feynman diagram for strings is depicted in figure 2. A Wick rotated $g$-loop $N$-string diagram is conformally equivalent to an $N$ punctured genus $g$ Riemann surface $\Sigma$. A $g$-loop $N$-string amplitude is given as an integral over the moduli 
space of $\Sigma$ as $[7,17]$

$$
\mathcal{A}_{N}^{(g)}=\left(i g_{s}\right)^{2 g-2+N} C \int[d T][\alpha d \theta][d \alpha] F_{N}^{(g)}
$$

where $\int[d T][\alpha d \theta][d \alpha]$ denotes the integration over the moduli parameters and $C$ is the combinatorial factor. The integrand $F_{N}^{(g)}$ is given as a path integral over the transverse variables $X^{i}, \psi^{i}, \bar{\psi}^{i}$ on the light-cone diagram. A light-cone diagram consists of cylinders which correspond to propagators of the closed string. On each cylinder one can introduce a complex coordinate

$$
\rho=\tau+i \sigma
$$

whose real part $\tau$ coincides with the Wick rotated light-cone time it and imaginary part $\sigma \sim \sigma+2 \pi \alpha_{r}$ parametrizes the closed string at each time. The $\rho$ 's on the cylinders are smoothly connected except at the interaction points and we get a complex coordinate $\rho$ on $\Sigma$. The path integral on the light-cone diagram is defined by using the metric

$$
d s^{2}=d \rho d \bar{\rho} .
$$

$\rho$ is not a good coordinate around the interaction points and the punctures, and the metric $(2.7)$ is not well-defined at these points. $F_{N}^{(g)}$ can be expressed in terms of correlation functions defined with a metric $d \hat{s}^{2}=2 \hat{g}_{z \bar{z}} d z d \bar{z}$ which is regular everywhere on the worldsheet, as

$$
\begin{aligned}
F_{N}^{(g)}= & (2 \pi)^{2} \delta\left(\sum_{r=1}^{N} p_{r}^{+}\right) \delta\left(\sum_{r=1}^{N} p_{r}^{-}\right) e^{-\frac{1}{2} \Gamma\left[\sigma ; \hat{g}_{z \bar{z}}\right]} \\
& \times \sum_{\text {spin structure }} \int\left[d X^{i} d \psi^{i} d \bar{\psi}^{i}\right]_{\hat{g}_{z \bar{z}}} e^{-S^{\mathrm{LC}}\left[X^{i}, \psi^{i}, \bar{\psi}^{i}\right]} \\
& \times \prod_{I=1}^{2 g-2+N}\left(\left|\partial^{2} \rho\left(z_{I}\right)\right|^{-\frac{3}{2}} T_{F}^{\mathrm{LC}}\left(z_{I}\right) \bar{T}_{F}^{\mathrm{LC}}\left(\bar{z}_{I}\right)\right) \prod_{r=1}^{N} V_{r}^{\mathrm{LC}}\left(Z_{r}, \bar{Z}_{r}\right) .
\end{aligned}
$$

Here $z$ is a complex coordinate of the Riemann surface and the coordinate $\rho$ becomes a function $\rho(z)$ of $z$ (see e.g. [18-20]). $S^{\mathrm{LC}}\left[X^{i}, \psi^{i}, \bar{\psi}^{i}\right]$ denotes the worldsheet action of the transverse variables and the path integral measure $\left[d X^{i} d \psi^{i} d \bar{\psi}^{i}\right]_{\hat{g}_{z \bar{z}}}$ is defined with the metric $d \hat{s}^{2}=2 \hat{g}_{z \bar{z}} d z d \bar{z}$. Since the integrand was defined by using the metric (2.7), we need the anomaly factor $e^{-\frac{1}{2} \Gamma\left[\sigma ; \hat{g}_{z \bar{z}}\right]}$, where

$$
\begin{aligned}
\sigma & =\ln \partial \rho \bar{\partial} \bar{\rho}-\ln \hat{g}_{z \bar{z}} \\
\Gamma\left[\sigma ; \hat{g}_{z \bar{z}}\right] & =-\frac{1}{4 \pi} \int d z \wedge d \bar{z} \sqrt{\hat{g}}\left(\hat{g}^{a b} \partial_{a} \sigma \partial_{b} \sigma+2 \hat{R} \sigma\right) .
\end{aligned}
$$

$z_{I}(I=1, \cdots, 2 g-2+N)$ denote the $z$-coordinates of the interaction points of the lightcone gauge Feynman diagram. $V_{r}^{\mathrm{LC}}$ denotes the vertex operator for the $r$-th external line inserted at $z=Z_{r}(r=1, \ldots, N)$. The right hand side of (2.8) does not depend on the choice of $\hat{g}_{z \bar{z}}$. 
As was demonstrated in [13], if all the external lines are in the (NS,NS) sector and the spin structure for the left and right fermions are both even, the term in the sum in (2.8) can be recast into a conformal gauge expression:

$$
\begin{aligned}
& \int\left[d X^{\mu} d \psi^{\mu} d \bar{\psi}^{\mu} d b d \bar{b} d c d \bar{c} d \beta d \bar{\beta} d \gamma d \bar{\gamma}\right]_{\hat{g}_{z \bar{z}}} e^{-S^{\mathrm{tot}}} \\
& \quad \times \prod_{K=1}^{6 g-6+2 N}\left[\oint_{C_{K}} \frac{d z}{\partial \rho} b_{z z}+\varepsilon_{K} \oint_{\bar{C}_{K}} \frac{d \bar{z}}{\bar{\partial} \bar{\rho}} b_{\bar{z} \bar{z}}\right]^{2 g-2+N} \prod_{I=1}^{N}\left[X\left(z_{I}\right) \bar{X}\left(\bar{z}_{I}\right)\right] \\
& \quad \times \prod_{r=1}^{N} V_{r}^{(-1,-1)}\left(Z_{r}, \bar{Z}_{r}\right) .
\end{aligned}
$$

Here $S^{\text {tot }}$ denotes the worldsheet action for the variables $X^{\mu}, \psi^{\mu}, \bar{\psi}^{\mu}(\mu=+,-, 1, \ldots, 8)$, ghosts and superghosts,

$$
X(z)=\left[c \partial \xi-e^{\phi} T_{F}+\frac{1}{4} \partial b \eta e^{2 \phi}+\frac{1}{4} b\left(2 \partial \eta e^{2 \phi}+\eta \partial e^{2 \phi}\right)\right](z)
$$

is the picture changing operator $(\mathrm{PCO}), \bar{X}(\bar{z})$ is its antiholomorphic counterpart and $T_{F}$ denotes the supercurrent for $\partial X^{\mu}, \psi^{\mu}$. The contours $C_{K}$ and $\varepsilon_{K}= \pm 1$ are chosen so that the antighost insertions correspond to the moduli parameters for the light-cone amplitudes. The vertex operator $V_{r}^{(-1,-1)}\left(Z_{r}, \bar{Z}_{r}\right)$ is defined as

$$
V_{r}^{(-1,-1)}\left(Z_{r}, \bar{Z}_{r}\right) \equiv c \bar{c} e^{-\phi-\bar{\phi}} V_{r}^{\mathrm{DDF}}\left(Z_{r}, \bar{Z}_{r}\right) .
$$

$V_{r}^{\mathrm{DDF}}\left(Z_{r}, \bar{Z}_{r}\right)$ is the supersymmetric DDF vertex operator given by

$V_{r}^{\mathrm{DDF}}\left(Z_{r}, \bar{Z}_{r}\right)=A_{-n_{1}}^{i_{1}(r)} \cdots \bar{A}_{-\bar{n}_{1}}^{\bar{i}_{1}(r)} \cdots B_{-s_{1}}^{j_{1}(r)} \cdots \bar{B}_{-\bar{s}_{1}}^{\bar{j}_{1}(r)} \cdots e^{-i p_{r}^{+} X^{-}-i\left(p_{r}^{-}-\frac{\mathcal{N}_{r}}{p_{r}^{+}}\right) X^{+}+i p_{r}^{i} X^{i}}\left(Z_{r}, \bar{Z}_{r}\right)$,

with the DDF operators $A_{-n}^{i(r)}, B_{-s}^{j(r)}$ for the $r$-th string defined as

$$
\begin{aligned}
& A_{-n}^{i(r)}=\oint_{Z_{r}} \frac{d \mathbf{z}}{2 \pi i} i D \mathcal{X}^{i} e^{-i \frac{n}{p_{r}^{+}} \mathcal{X}_{L}^{+}}(\mathbf{z}), \\
& B_{-s}^{i(r)}=\oint_{Z_{r}} \frac{d \mathbf{z}}{2 \pi i} \frac{D \mathcal{X}^{+}}{\left(i p_{r}^{+} \partial \mathcal{X}^{+}\right)^{\frac{1}{2}}} D \mathcal{X}^{i} e^{-i \frac{s}{p_{r}^{+}} \mathcal{X}_{L}^{+}}(\mathbf{z}),
\end{aligned}
$$

and $\bar{A}_{-n}^{i(r)}, \bar{B}_{-s}^{i(r)}$ are similarly given for the antiholomorphic sector. Here we use the notation

$$
\begin{aligned}
\mathcal{N}_{r} & \equiv \sum_{k} n_{k}+\sum_{l} s_{l}=\sum_{\bar{k}} \bar{n}_{\bar{k}}+\sum_{\bar{l}} \bar{s}_{\bar{l}}, \\
\mathcal{X}^{\mu}(\mathbf{z}, \overline{\mathbf{z}}) & =X^{\mu}(z, \bar{z})+i \theta \psi^{\mu}(z)+i \bar{\theta} \bar{\psi}^{\mu}(\bar{z})+\theta \bar{\theta} F^{\mu}, \\
D & \equiv \frac{\partial}{\partial \theta}+\theta \frac{\partial}{\partial z} .
\end{aligned}
$$

$\mathbf{z}=(z, \theta)$ denotes the superspace coordinate on the worldsheet and $\mathcal{X}_{L}^{+}$denotes the leftmoving part of the superfield $\mathcal{X}^{+} .{ }^{1}$ We take the vertex operators to satisfy the on-shell

\footnotetext{
${ }^{1}$ Although $\mathcal{X}_{L}^{+}$is not a well-defined quantity, it is used as a short-hand notation to express the vertex operator (2.13), which is well-defined.
} 
condition

$$
\frac{1}{2}\left(-2 p_{r}^{+} p_{r}^{-}+p_{r}^{i} p_{r}^{i}\right)+\mathcal{N}_{r}=\frac{1}{2}
$$

$V_{r}^{\mathrm{DDF}}\left(Z_{r}, \bar{Z}_{r}\right)$ turns out to be a weight $\left(\frac{1}{2}, \frac{1}{2}\right)$ primary field made from $X^{\mu}, \psi^{\mu}, \bar{\psi}^{\mu}$. Therefore $V_{r}^{(-1,-1)}\left(Z_{r}, \bar{Z}_{r}\right)$ is an on-shell vertex operator in $(-1,-1)$ picture. It is easy to see that the expression of the amplitude (2.5) given as an integral of (2.10) is BRST invariant.

One way to derive the expression (2.10) is as follows [13]. Using a nilpotent fermionic charge, it is possible to show that the right hand side of (2.10) is equal to

$$
\begin{aligned}
\int[ & \left.d X^{\mu} d \psi^{\mu} d \bar{\psi}^{\mu} d b d \bar{b} d c d \bar{c} d \beta d \bar{\beta} d \gamma d \bar{\gamma}\right]_{\hat{g}_{z \bar{z}}} e^{-S^{\mathrm{tot}}} \\
& \times \prod_{K=1}^{6 g-6+2 N}\left[\oint_{C_{K}} \frac{d z}{\partial \rho} b_{z z}+\varepsilon_{K} \oint_{\bar{C}_{K}} \frac{d \bar{z}}{\bar{\partial} \bar{\rho}} b_{\bar{z} \bar{z}}\right]^{2 g-2+N} \prod_{I=1}^{N}\left[e^{\phi} T_{\mathrm{F}}^{\mathrm{LC}}\left(z_{I}\right) e^{\bar{\phi}} \bar{T}_{\mathrm{F}}^{\mathrm{LC}}\left(\bar{z}_{I}\right)\right] \\
& \times \prod_{r=1}^{N}\left[c \bar{c} e^{-\phi-\bar{\phi}} V_{r}^{\mathrm{DDF}}\left(Z_{r}, \bar{Z}_{r}\right)\right]
\end{aligned}
$$

In this form, the path integral factorizes into the contributions from $X^{\mu}, \psi^{\mu}, \bar{\psi}^{\mu}$, ghosts and superghosts. Each of these contributions is calculated by taking $\hat{g}_{z \bar{z}}$ to be the Arakelov metric $g_{z \bar{z}}^{\mathrm{A}}[21]$. In the matter sector, integration over the longitudinal variables yields

$$
\begin{aligned}
& \int\left[d X^{ \pm} d \psi^{ \pm} d \bar{\psi}^{ \pm}\right]_{g_{z \bar{z}}^{\mathrm{A}}} e^{-S^{ \pm}} \prod_{r=1}^{N} V_{r}^{\mathrm{DDF}}\left(Z_{r}, \bar{Z}_{r}\right) \\
& =(2 \pi)^{2} \delta\left(\sum_{r=1}^{N} p_{r}^{-}\right) \delta\left(\sum_{r=1}^{N} p_{r}^{+}\right) Z_{X^{ \pm}}\left[g_{z \bar{z}}^{\mathrm{A}}\right] Z_{\psi^{ \pm}}\left[g_{z \bar{z}}^{\mathrm{A}}\right] \prod_{r=1}^{N}\left[\frac{1}{\alpha_{r}} e^{-\operatorname{Re} \bar{N}_{00}^{r r}} V_{r}^{\mathrm{LC}}\left(Z_{r}, \bar{Z}_{r}\right)\right]
\end{aligned}
$$

where

$$
\begin{aligned}
Z_{X^{ \pm}}\left[g_{z \bar{z}}\right] & =\left(\frac{\operatorname{det}^{\prime}\left(-g^{z \bar{z}} \partial_{z} \partial_{\bar{z}}\right)}{\int d^{2} z \sqrt{g}}\right)^{-1}, \\
Z_{\psi^{ \pm}}\left[g_{z \bar{z}}\right] & =\left(\frac{\operatorname{det}^{\prime}\left(-g^{z \bar{z}} \partial_{z} \partial_{\bar{z}}\right)}{\operatorname{det} \operatorname{Im} \Omega \int d^{2} z \sqrt{g}}\right)^{-\frac{1}{2}} \vartheta\left[\alpha_{\mathrm{L}}\right](0) \vartheta\left[\alpha_{\mathrm{R}}\right](0)^{*} \\
\bar{N}_{00}^{r r} & =\lim _{z \rightarrow Z_{r}}\left[\frac{\rho\left(z_{I^{(r)}}\right)-\rho(z)}{\alpha_{r}}+\ln \left(z-Z_{r}\right)\right] .
\end{aligned}
$$

Here $\vartheta[\alpha]$ denotes the theta function with characteristic $\alpha$ and $\Omega$ is the period matrix. $\alpha_{\mathrm{L}}$ and $\alpha_{\mathrm{R}}$ denote the characteristics corresponding to the spin structures of the left- and right-moving fermions respectively. $Z_{X^{ \pm}}\left[g_{z \bar{z}}\right]$ and $Z_{\psi^{ \pm}}\left[g_{z \bar{z}}\right]$ are respectively the partition functions of the free variables $X^{ \pm}$and $\psi^{ \pm}, \bar{\psi}^{ \pm}$on the worldsheet endowed with the metric $d s^{2}=2 g_{z \bar{z}} d z d \bar{z} . z_{I^{(r)}}$ denotes the interaction point at which the $r$-th string interacts. The 
contributions from the ghosts and superghosts are given as

$$
\begin{gathered}
\int[d b d \bar{b} d c d \bar{c}]_{g_{z \bar{z}}^{\mathrm{A}}} e^{-S^{b c}} \prod_{r=1}^{N} c \bar{c}\left(Z_{r}, \bar{Z}_{r}\right) \prod_{K=1}^{6 g-6+2 N}\left[\oint_{C_{K}} \frac{d z}{\partial \rho} b_{z z}+\varepsilon_{K} \oint_{\bar{C}_{K}} \frac{d \bar{z}}{\bar{\partial} \bar{\rho}} b_{\bar{z} \bar{z}}\right] \\
=\left(Z_{X^{ \pm}}\left[g_{z \bar{z}}^{\mathrm{A}}\right]\right)^{-1} e^{-\Gamma\left[\sigma ; g_{z \bar{z}}^{\mathrm{A}}\right]} \prod_{r=1}^{N}\left(\alpha_{r} e^{2 \operatorname{Re} \bar{N}_{00}^{r r}}\right), \\
\int[d \beta d \gamma d \bar{\beta} d \bar{\gamma}]_{g_{z \bar{z}}^{\mathrm{A}}} e^{-S_{\beta \gamma}} \prod_{I=1}^{2 g-2+N}\left[e^{\phi}\left(z_{I}\right) e^{\bar{\phi}}\left(\bar{z}_{I}\right)\right] \prod_{r=1}^{N}\left[e^{-\phi}\left(Z_{r}\right) e^{-\bar{\phi}}\left(\bar{Z}_{r}\right)\right] \\
=\left(Z_{\psi^{ \pm}}\left[g_{z \bar{z}}^{\mathrm{A}}\right]\right)^{-1} e^{\frac{1}{2} \Gamma\left[\sigma ; g_{z \bar{z}}^{\mathrm{A}}\right]} \prod_{r=1}^{N} e^{-\operatorname{Re} \bar{N}_{00}^{r r}} \prod_{I=1}^{2 g-2+N}\left|\partial^{2} \rho\left(z_{I}\right)\right|^{-\frac{3}{2}} .
\end{gathered}
$$

Substituting eqs. (2.18), (2.20), (2.21) into (2.17), we can easily see that (2.10) is equal to $(2.8)$.

\subsection{Dimensional regularization}

The amplitudes of superstring theory were calculated using the first-quantized formalism in [22] in which an expression using the PCO's was given. The expression (2.10) is a special case of the one in [22], where the PCO's are placed at the interaction points of the light-cone Feynman diagram. ${ }^{2}$

Unfortunately, the amplitude (2.5) given as an integral of (2.10) or (2.8) is not welldefined. (2.8) diverges when some of the interaction points collide, because $T_{\mathrm{F}}^{\mathrm{LC}}(z)$ has the OPE

$$
T_{\mathrm{F}}^{\mathrm{LC}}\left(z_{I}\right) T_{\mathrm{F}}^{\mathrm{LC}}\left(z_{J}\right) \sim \frac{2}{\left(z_{I}-z_{J}\right)^{3}}+\cdots,
$$

which makes the integral (2.5) ill-defined. This kind of divergence is called the contact term divergence.

Accordingly, the conformal gauge expression (2.10) suffers from the so-called spurious singularity. The holomorphic part of the correlation function of the superghost system has the form

$$
\begin{aligned}
& \left\langle\prod_{I=1}^{2 g-2+N} e^{\phi}\left(z_{I}\right) \prod_{r=1}^{N} e^{-\phi}\left(Z_{r}\right)\right\rangle \\
& \sim\left[\vartheta\left[\alpha_{\mathrm{L}}\right]\left(-\sum_{r} \int_{P_{0}}^{Z_{r}} \omega+\sum_{I} \int_{P_{0}}^{z_{I}} \omega-2 \int_{P_{0}}^{\triangle} \omega\right)\right]^{-1} \\
& \quad \times \frac{\prod_{I, r} E\left(z_{I}, Z_{r}\right)}{\prod_{I<J} E\left(z_{I}, z_{J}\right) \prod_{r<s} E\left(Z_{r}, Z_{s}\right)} \frac{\prod_{r} \sigma^{2}\left(Z_{r}\right)}{\prod_{I} \sigma^{2}\left(z_{I}\right)} .
\end{aligned}
$$

Here $\omega$ is the canonical basis of the holomorphic 1-forms, $\triangle$ is the Riemann class, $E(z, w)$ is the prime form of the surface and $\sigma(z)$ is a holomorphic $\frac{g}{2}$ form with no zeros or poles. The base point $P_{0}$ is an arbitrary point on the surface. ${ }^{3}$ This correlation function diverges when

\footnotetext{
${ }^{2}$ Notice that in the light-cone setup, the positions of the PCO's have the fixed coordinate in the coordinate patch on the surface and we do not need $\partial \xi$ terms.

${ }^{3}$ For the mathematical background relevant for string perturbation theory, we refer the reader to [19].
} 
1. Some of $z_{I}$ collide.

2. $\vartheta\left[\alpha_{\mathrm{L}}\right]\left(-\sum_{r} \int_{P_{0}}^{Z_{r}} \omega+\sum_{I} \int_{P_{0}}^{z_{I}} \omega-2 \int_{P_{0}}^{\triangle} \omega\right)=0$.

It also diverges when some of $Z_{r}$ collide, but such singularities are at the boundary of moduli space of the punctured Riemann surface. The singularities given above are called the spurious singularities. The first type of singularity corresponds to the contact term divergence mentioned above. The second type of singularity is due to existence of zero modes of $\gamma$. Singularities of this kind do not arise in our case. Since $Z_{r}(r=1, \ldots N)$ and $z_{I}(I=1, \ldots, 2 g-2+N)$ are the poles and the zeros of the meromorphic one-form $\partial \rho(z) d z$ respectively, $\sum_{I} z_{I}-\sum_{r} Z_{r}$ is a canonical divisor on the surface. Therefore we obtain

$$
-\sum_{r=1}^{N} \int_{P_{0}}^{Z_{r}} \omega+\sum_{I=1}^{2 g-2+N} \int_{P_{0}}^{z_{I}} \omega \equiv 2 \int_{P_{0}}^{\triangle} \omega \quad\left(\bmod \mathbb{Z}^{g}+\mathbb{Z}^{g} \Omega\right),
$$

where $\Omega$ is the period matrix. This yields

$$
\left[\vartheta\left[\alpha_{\mathrm{L}}\right]\left(-\sum_{r} \int_{P_{0}}^{Z_{r}} \omega+\sum_{I} \int_{P_{0}}^{z_{I}} \omega-2 \int_{P_{0}}^{\triangle} \omega\right)\right]^{-1}=\left[\vartheta\left[\alpha_{\mathrm{L}}\right](0)\right]^{-1},
$$

which is included in the factor $\left(Z_{\psi^{ \pm}}\right)^{-1}$ in $(2.21)$. $\left[\vartheta\left[\alpha_{\mathrm{L}}\right](0)\right]^{-1}$ may become singular at some points in the moduli space, but the $\left[\vartheta\left[\alpha_{\mathrm{L}}\right](0)\right]^{-1}$ cancels the factor $\vartheta\left[\alpha_{\mathrm{L}}\right](0)$ from the partition function $Z_{\psi^{ \pm}}$of $\psi^{ \pm}$and the whole amplitude is free from this type of singularity.

Therefore, in order to make the amplitudes given in the previous subsection welldefined, we should deal with the contact term divergences. In our previous works, we employ the dimensional regularization to do so. Let us summarize the results:

- One can formulate the light-cone gauge superstring field theory in $d \neq 10$ dimensional space time. The amplitudes are given in the form (2.5) with

$$
\begin{aligned}
F_{N}^{(g)}= & (2 \pi)^{2} \delta\left(\sum_{r=1}^{N} p_{r}^{+}\right) \delta\left(\sum_{r=1}^{N} p_{r}^{-}\right) e^{-\frac{d-2}{16} \Gamma\left[\sigma ; \hat{g}_{z \bar{z}}\right]} \\
\times \sum_{\text {spin structure }} \int\left[d X^{i} d \psi^{i} d \bar{\psi}^{i}\right]_{\hat{g}_{z \bar{z}}} e^{-S^{\mathrm{LC}}\left[X^{i}, \psi^{i}, \bar{\psi}^{i}\right]} & \\
& \times \prod_{I=1}^{2 g-2+N}\left(\left|\partial^{2} \rho\left(z_{I}\right)\right|^{-\frac{3}{2}} T_{F}^{\mathrm{LC}}\left(z_{I}\right) \bar{T}_{F}^{\mathrm{LC}}\left(\bar{z}_{I}\right)\right) \prod_{r=1}^{N} V_{r}^{\mathrm{LC}}
\end{aligned}
$$

Taking $d$ to be large and negative, the factor $e^{-\frac{d-2}{16} \Gamma\left[\sigma ; \hat{g}_{z \bar{z}}\right]}$ tame the contact term divergences.

- More generally we can regularize the divergences by taking the worldsheet superconformal field theory to be the one with central charge $c \neq 12$. One convenient choice of the worldsheet theory is the one in a linear dilaton background $\Phi=-i Q X^{1}$, with 
a real constant $Q$. The worldsheet action of $X^{1}$ and its fermionic partners $\psi^{1}, \bar{\psi}^{1}$ on a worldsheet with metric $d \hat{s}^{2}=2 \hat{g}_{z \bar{z}} d z d \bar{z}$ becomes

$$
\begin{aligned}
S\left[X^{1}, \psi^{1}, \bar{\psi}^{1} ; \hat{g}_{z \bar{z}}\right]= & \frac{1}{8 \pi} \int d z \wedge d \bar{z} \sqrt{\hat{g}}\left(\hat{g}^{a b} \partial_{a} X^{1} \partial_{b} X^{1}-2 i Q \hat{R} X^{1}\right) \\
& +\frac{1}{4 \pi} \int d z \wedge d \bar{z} i\left(\psi^{1} \bar{\partial} \psi^{1}+\bar{\psi}^{1} \partial \bar{\psi}^{1}\right),
\end{aligned}
$$

The amplitude is expressed in the form (2.26) with

$$
d=10-8 Q^{2} .
$$

It was shown in [14] that (with the Feynman $i \varepsilon$ ) by taking $Q^{2}>10$, the amplitudes become finite.

- We can define the amplitudes as analytic functions of $Q^{2}$ and take the limit $Q \rightarrow 0$ to obtain those in $d=10$. In order to study the limit, it is useful to recast the expression (2.26) into the conformal gauge one [13]

$$
\begin{aligned}
\int[ & \left.d X^{\mu} d \psi^{\mu} d \bar{\psi}^{\mu} d b d \bar{b} d c d \bar{c} d \beta d \bar{\beta} d \gamma d \bar{\gamma}\right]_{g_{z \bar{z}}^{\mathrm{A}}} e^{-S^{\mathrm{tot}}} \\
\quad \times & \prod_{K=1}^{6 g-6+2 N}\left[\oint_{C_{K}} \frac{d z}{\partial \rho} b_{z z}+\varepsilon_{K} \oint_{\bar{C}_{K}} \frac{d \bar{z}}{\bar{\partial} \bar{\rho}} b_{\bar{z} \bar{z}}\right]^{2 g-2+N} \prod_{I=1}^{N}\left[X\left(z_{I}\right) \bar{X}\left(\bar{z}_{I}\right)\right] \\
& \times \prod_{r} e^{-\frac{i Q^{2}}{\alpha_{r}} \mathcal{X}^{+}}\left(\hat{\tilde{\mathbf{z}}}_{I^{(r)}}, \hat{\tilde{\mathbf{z}}}_{I^{(r)}}\right) \prod_{r=1}^{N}\left[V_{r}^{(-1,-1)}\left(Z_{r}, \bar{Z}_{r}\right)\right]
\end{aligned}
$$

which looks quite similar to the critical case $(2.10) .{ }^{4}$ The crucial difference is that the worldsheet theory for the longitudinal variables $X^{ \pm}, \psi^{ \pm}, \bar{\psi}^{ \pm}$is a superconformal field theory called the supersymmetric $X^{ \pm}$CFT, which has the central charge

$$
c=3+12 Q^{2} .
$$

With this CFT, we can construct a nilpotent BRST charge. Using this expression, one can show that the amplitudes in the limit $Q \rightarrow 0$ coincide with those given by the Sen-Witten prescription [23], if the latter exists.

\subsection{The problems with odd spin structure}

The light-cone gauge amplitudes can be defined and calculated for odd spin structure, and we get the expression (2.5) with the integrand given by (2.8). The correlation functions

${ }^{4}$ Notice that the expression here is different from the one in [13] where the operators

$$
\oint_{z_{I^{(r)}}} \frac{d \mathbf{z}}{2 \pi i} \mathcal{S}\left(\mathbf{z}, Z_{r}\right) \oint_{\bar{z}_{I^{(r)}}} \frac{d \overline{\mathbf{z}}}{2 \pi i} \overline{\mathcal{S}}\left(\overline{\mathbf{z}}, \bar{Z}_{r}\right)
$$

are inserted in place of $e^{-\frac{i Q^{2}}{\alpha_{r}} \mathcal{X}^{+}}\left(\hat{\tilde{\mathbf{z}}}_{I^{(r)}}, \hat{\tilde{\mathbf{z}}}_{I^{(r)}}\right)$. The properties of operators of this kind with operator valued $\operatorname{arguments} \hat{\tilde{\mathbf{z}}}_{I}, \hat{\tilde{\mathbf{z}}}_{I}$ are explained in appendix A. 
of free fermions on higher genus Riemann surfaces are given in appendix B. Only the amplitudes with enough fermions from the vertex operators and $T_{\mathrm{F}}$ insertions to soak up the zero modes are nonvanishing. However, we have a problem in rewriting the light-cone gauge expression (2.8) into the BRST invariant one (2.10), if we proceed as in the previous section. If $\alpha$ corresponds to an odd spin structure,

$$
\vartheta[\alpha](0)=0
$$

The correlation function (2.21) of the $\beta, \gamma$ system diverges because it involves factors

$$
(\vartheta[\alpha](0))^{-1}
$$

coming from $\left(Z_{\psi^{ \pm}}\right)^{-1}$ on the right hand side of (2.21). On the other hand, the partition function (2.19) of the $\psi^{ \pm}$variables involves factors

$$
\vartheta[\alpha](0)
$$

which cancel the divergent contribution from the $\beta, \gamma$ system. Therefore we need to make sense out of the combination

$$
0 \times \infty
$$

to obtain the BRST invariant expression corresponding to the light-cone gauge amplitudes.

\section{Odd spin structure}

The problem mentioned at the end of the previous section can be avoided by considering the amplitudes with insertions of $\psi^{+}, \psi^{-}$and $\delta(\beta), \delta(\gamma)$. We would like to show that such insertions can be realized in a BRST invariant way, if we consider the conformal gauge amplitudes taking some of the vertex operators to have 0 or -2 picture, when all the external lines are in the (NS,NS) sector.

\subsection{Multiloop amplitudes}

Let us consider the case where the spin structure $\alpha_{\mathrm{L}}$ for the left-moving fermions is odd and $\alpha_{\mathrm{R}}$ for the right-moving fermions is even. The case where $\alpha_{\mathrm{L}}$ is even and $\alpha_{\mathrm{R}}$ is odd or both of $\alpha_{\mathrm{L}}$ and $\alpha_{\mathrm{R}}$ are odd can be dealt with in the same way. We would like to show that the term

$$
\begin{aligned}
\int\left[d X^{i} d \psi^{i} d \bar{\psi}^{i}\right]_{\hat{g}_{z \bar{z}}} & e^{-S^{\mathrm{LC}}\left[X^{i}, \psi^{i}, \bar{\psi}^{i}\right]} \\
& \times \prod_{I=1}^{2 g-2+N}\left(\left|\partial^{2} \rho\left(z_{I}\right)\right|^{-\frac{3}{2}} T_{F}^{\mathrm{LC}}\left(z_{I}\right) \bar{T}_{F}^{\mathrm{LC}}\left(\bar{z}_{I}\right)\right) \prod_{r=1}^{N} V_{r}^{\mathrm{LC}}\left(Z_{r}, \bar{Z}_{r}\right),
\end{aligned}
$$


in the sum in (2.8) corresponding to such a spin structure can be recast into a conformal gauge expression

$$
\begin{aligned}
& \int\left[d X^{\mu} d \psi^{\mu} d \bar{\psi}^{\mu} d b d \bar{b} d c d \bar{c} d \beta d \bar{\beta} d \gamma d \bar{\gamma}\right]_{g_{z \bar{z}}^{\mathrm{A}}} e^{-S^{\mathrm{tot}}} \\
& \quad \times \prod_{K=1}^{6 g-6+2 N}\left[\oint_{C_{K}} \frac{d z}{\partial \rho} b_{z z}+\varepsilon_{K} \oint_{\bar{C}_{K}} \frac{d \bar{z}}{\bar{\partial} \bar{\rho}} b_{\bar{z} \bar{z}}\right] \prod_{I}\left[X\left(z_{I}\right) \bar{X}\left(\bar{z}_{I}\right)\right] \\
& \quad \times V_{1}^{(-2,-1)}\left(Z_{1}, \bar{Z}_{1}\right) V_{2}^{(0,-1)}\left(Z_{2}, \bar{Z}_{2}\right) \prod_{r=3}^{N}\left[V_{r}^{(-1,-1)}\left(Z_{r}, \bar{Z}_{r}\right)\right]
\end{aligned}
$$

up to a numerical factor. As we will see, the expression (3.2) is well-defined and free from the combination $0 \times \infty$. Here $V_{r}^{(-1,-1)}\left(Z_{r}, \bar{Z}_{r}\right)$ is the $(-1,-1)$ picture vertex operator defined in $(2.12)$ and $V_{1}^{(-2,-1)}\left(Z_{1}, \bar{Z}_{1}\right), V_{2}^{(0,-1)}\left(Z_{2}, \bar{Z}_{2}\right)$ are given by

$$
\begin{aligned}
V_{1}^{(-2,-1)}\left(Z_{1}, \bar{Z}_{1}\right) & =-\frac{2}{p_{1}^{+}} c \bar{c} e^{-2 \phi} e^{-\bar{\phi}} \psi^{+} V_{1}^{\mathrm{DDF}}\left(Z_{1}, \bar{Z}_{1}\right) \\
V_{2}^{(0,-1)}\left(Z_{2}, \bar{Z}_{2}\right) & =\left[-c \bar{c} e^{-\bar{\phi}} \oint_{Z_{2}} \frac{d z}{2 \pi i} T_{\mathrm{F}}(z)+\frac{1}{4} \bar{c} \gamma e^{-\bar{\phi}}\right] V_{2}^{\mathrm{DDF}}\left(Z_{2}, \bar{Z}_{2}\right),
\end{aligned}
$$

which satisfy

$$
\begin{aligned}
X V_{1}^{(-2,-1)}\left(Z_{1}, \bar{Z}_{1}\right) & =V_{1}^{(-1,-1)}\left(Z_{1}, \bar{Z}_{1}\right), \\
X V_{2}^{(-1,-1)}\left(Z_{2}, \bar{Z}_{2}\right) & =V_{2}^{(0,-1)}\left(Z_{2}, \bar{Z}_{2}\right), \\
Q_{\mathrm{B}} V_{1}^{(-2,-1)}\left(Z_{1}, \bar{Z}_{1}\right) & =0
\end{aligned}
$$

where $X$ is the picture changing operator (2.11) and $Q_{\mathrm{B}}$ denotes the BRST charge (C.12). Since $V^{\mathrm{DDF}}(Z, \bar{Z})$ is expressed as $(2.13), V_{2}^{(0,-1)}\left(Z_{2}, \bar{Z}_{2}\right)$ can be rewritten as

$$
\begin{aligned}
& V_{2}^{(0,-1)}\left(Z_{2}, \bar{Z}_{2}\right) \\
& \begin{aligned}
= & -c \bar{c} e^{-\bar{\phi}} A_{-n_{1}}^{i_{1}(2)} \cdots \bar{A}_{-\bar{n}_{1}}^{\bar{i}_{1}(2)} \cdots B_{-s_{1}}^{j_{1}(2)} \cdots \bar{B}_{-\bar{s}_{1}}^{\bar{j}_{1}(2)} \cdots \\
& \quad \times \frac{1}{2}\left[p_{2}^{+} \psi^{-}+\left(p_{2}^{-}-\frac{\mathcal{N}_{2}}{p_{2}^{+}}\right) \psi^{+}-p_{2}^{i} \psi^{i}\right] e^{-i p_{2}^{+} X^{-}-i\left(p_{2}^{-}-\frac{\mathcal{N}_{2}}{p_{2}^{+}}\right) X^{+}+i p_{2}^{i} X^{i}} \\
& \quad+\frac{1}{4} \bar{c} \gamma e^{-\bar{\phi}} V_{2}^{\mathrm{DDF}}\left(Z_{2}, \bar{Z}_{2}\right) \\
= & -\frac{1}{2} c \bar{c} e^{-\bar{\phi}} p_{2}^{+}: \psi^{-} V_{2}^{\mathrm{DDF}}\left(Z_{2}, \bar{Z}_{2}\right):+\cdots
\end{aligned}
\end{aligned}
$$

where the ellipses in the last line denote the terms which do not involve $\psi^{-}$.

$V_{1}^{(-2,-1)}\left(Z_{1}, \bar{Z}_{1}\right), V_{2}^{(0,-1)}\left(Z_{2}, \bar{Z}_{2}\right)$ can be considered to be the BRST invariant vertex operators in $(-2,-1),(0,-1)$ pictures respectively. It is straightforward to define vertex operators $V^{(-1,-2,)}, V^{(-1,0)}$, or $V^{(-2,-2)}, V^{(0,0)}$ which can be used to express the amplitudes for the cases of the other spin structures mentioned above. 
It is possible to show that (3.2) is equal to

$$
\begin{aligned}
& \int\left[d X^{\mu} d \psi^{\mu} d \bar{\psi}^{\mu} d b d \bar{b} d c d \bar{c} d \beta d \bar{\beta} d \gamma d \bar{\gamma}\right]_{g_{z \bar{z}}^{\mathrm{A}}} e^{-S^{\mathrm{tot}}} \\
& \quad \times \prod_{K=1}^{6 g-6+2 N}\left[\oint_{C_{K}} \frac{d z}{\partial \rho} b_{z z}+\varepsilon_{K} \oint_{\bar{C}_{K}} \frac{d \bar{z}}{\bar{\partial} \bar{\rho}} b_{\bar{z} \bar{z}}\right] \prod_{I}\left[e^{\phi} T_{F}^{\mathrm{LC}}\left(z_{I}\right) e^{\bar{\phi}} \bar{T}_{F}^{\mathrm{LC}}\left(\bar{z}_{I}\right)\right] \\
& \quad \times V_{1}^{(-2,-1)}\left(Z_{1}, \bar{Z}_{1}\right) V_{2}^{(0,-1)}\left(Z_{2}, \bar{Z}_{2}\right) \prod_{r=3}^{N}\left[V_{r}^{(-1,-1)}\left(Z_{r}, \bar{Z}_{r}\right)\right]
\end{aligned}
$$

A poof of this fact can be found in appendix C.2. Therefore, in order to show that (3.1) is proportional to (3.2), we evaluate (3.7) and prove that it is proportional to (3.1). In (3.7), we can replace $V_{2}^{(0,-1)}\left(Z_{2}, \bar{Z}_{2}\right)$ by

$$
-\frac{1}{2} c \bar{c} e^{-\bar{\phi}} p_{2}^{+}: \psi^{-} V_{2}^{\mathrm{DDF}}:\left(Z_{2}, \bar{Z}_{2}\right)
$$

because only this term can soak up the zero mode of $\psi^{-}$. After such a replacement, (3.7) factorizes into contributions from the ghosts, superghosts, longitudinal modes and the transverse modes. The parts of the transverse variables and ghosts are the same as those in (2.17) and we can use (2.20) to evaluate the latter. In this case, the correlation function of the longitudinal variables is modified from (2.18) into

$$
\begin{aligned}
\int & {\left[d X^{ \pm} d \psi^{ \pm} d \bar{\psi}^{ \pm}\right]_{g_{z \bar{z}}^{\mathrm{A}}} e^{-S^{ \pm}} \psi^{+}\left(Z_{1}\right) \psi^{-}\left(Z_{2}\right) \prod_{r=1}^{N} V_{r}^{\mathrm{DDF}}\left(Z_{r}, \bar{Z}_{r}\right) } \\
= & (2 \pi)^{2} \delta\left(\sum_{r=1}^{N} p_{r}^{-}\right) \delta\left(\sum_{r=1}^{N} p_{r}^{+}\right) Z_{X^{ \pm}}\left[g_{z \bar{z}}^{\mathrm{A}}\right]\left(\frac{\operatorname{det}^{\prime}\left(-g^{\mathrm{A} z \bar{z}} \partial_{z} \partial_{\bar{z}}\right)}{\operatorname{det} \operatorname{Im} \Omega \int d^{2} z \sqrt{g^{\mathrm{A}}}}\right)^{-\frac{1}{2}} \vartheta\left[\alpha_{\mathrm{R}}\right](0)^{*} \\
& \times h_{\alpha_{\mathrm{L}}}\left(Z_{1}\right) h_{\alpha_{\mathrm{L}}}\left(Z_{2}\right) \prod_{r=1}^{N}\left[\frac{1}{\alpha_{r}} e^{-\operatorname{Re} \bar{N}_{00}^{r r}} V_{r}^{\mathrm{LC}}\left(Z_{r}, \bar{Z}_{r}\right)\right]
\end{aligned}
$$

and that of the superghosts is evaluated to be

$$
\begin{aligned}
\int & {[d \beta d \gamma d \bar{\beta} d \bar{\gamma}]_{g_{z \bar{z}}^{\mathrm{A}}} e^{-S_{\beta \gamma}} e^{-2 \phi}\left(Z_{1}\right) \prod_{I}\left[e^{\phi}\left(z_{I}\right) e^{\bar{\phi}}\left(\bar{z}_{I}\right)\right] \prod_{r=3}^{N} e^{-\phi}\left(Z_{r}\right) \prod_{r=1}^{N} e^{-\bar{\phi}}\left(\bar{Z}_{r}\right) } \\
\propto & \left(\frac{\operatorname{det}^{\prime}\left(-g^{\mathrm{A} z \bar{z}} \partial_{z} \partial_{\bar{z}}\right)}{\operatorname{det} \operatorname{Im} \Omega \int d^{2} z \sqrt{g^{\mathrm{A}}}}\right)^{\frac{1}{2}} \\
\times & \left.\times \vartheta\left[\alpha_{L}\right]\left(-\sum_{r=1}^{N} \int_{P_{0}}^{Z_{r}} \omega+\sum_{I} \int_{P_{0}}^{z_{I}} \omega-2 \int_{P_{0}}^{\triangle}+\int_{Z_{1}}^{Z_{2}} \omega\right)\right]^{-1} \\
\times & {\left[\vartheta\left[\alpha_{R}\right]\left(-\sum_{r=1}^{N} \int_{P_{0}}^{Z_{r}} \omega+\sum_{I} \int_{P_{0}}^{z_{I}} \omega-2 \int_{P_{0}}^{\triangle} \omega\right)^{*}\right]^{-1} } \\
& \times\left|\frac{\prod_{I, r} E\left(z_{I}, Z_{r}\right)}{\prod_{I<J} E\left(z_{I}, z_{J}\right) \prod_{r<s} E\left(Z_{r}, Z_{s}\right)} \frac{\prod_{r} \sigma^{2}\left(Z_{r}\right)}{\prod_{I} \sigma^{2}\left(z_{I}\right)}\right|^{2} e^{-12 S}
\end{aligned}
$$




$$
\begin{aligned}
& \times \frac{\prod_{r=3}^{N} E\left(Z_{2}, Z_{r}\right) \prod_{I} E\left(z_{I}, Z_{1}\right)}{\prod_{I} E\left(z_{I}, Z_{2}\right) \prod_{r=3}^{N} E\left(Z_{1}, Z_{r}\right)} \cdot \frac{\sigma^{2}\left(Z_{1}\right)}{\sigma^{2}\left(Z_{2}\right)} \cdot E\left(Z_{1}, Z_{2}\right) \\
& \propto \frac{\alpha_{1}}{\alpha_{2}}\left(\frac{\operatorname{det}^{\prime}\left(-g^{\mathrm{A} z \bar{z}} \partial_{z} \partial_{\bar{z}}\right)}{\operatorname{det} \operatorname{Im} \Omega \int d^{2} z \sqrt{g^{\mathrm{A}}}}\right)^{\frac{1}{2}}\left|\frac{\prod_{I, r} E\left(z_{I}, Z_{r}\right)}{\prod_{I<J} E\left(z_{I}, z_{J}\right) \prod_{r<s} E\left(Z_{r}, Z_{s}\right)} \frac{\prod_{r} \sigma^{2}\left(Z_{r}\right)}{\prod_{I} \sigma^{2}\left(z_{I}\right)}\right|^{2} e^{-12 S} \\
& \times \frac{1}{h_{\alpha_{\mathrm{L}}}\left(Z_{1}\right) h_{\alpha_{\mathrm{L}}}\left(Z_{2}\right) \vartheta\left[\alpha_{\mathrm{R}}\right](0)^{*}} \\
& =\frac{\alpha_{1}}{\alpha_{2}}\left(\frac{\operatorname{det}^{\prime}\left(-g^{\mathrm{A} z \bar{z}} \partial_{z} \partial_{\bar{z}}\right)}{\operatorname{det} \operatorname{Im} \Omega \int d^{2} z \sqrt{g^{\mathrm{A}}}}\right)^{\frac{1}{2}} e^{\frac{1}{2} \Gamma\left[\sigma ; g_{z \bar{z}}^{\mathrm{A}}\right]} \prod_{r} e^{-\operatorname{Re} \bar{N}_{00}^{r r}} \prod_{I}\left|\partial^{2} \rho\left(z_{I}\right)\right|^{-\frac{3}{2}} \\
& \times \frac{1}{h_{\alpha_{\mathrm{L}}}\left(Z_{1}\right) h_{\alpha_{\mathrm{L}}}\left(Z_{2}\right) \vartheta\left[\alpha_{\mathrm{R}}\right](0)^{*}} .
\end{aligned}
$$

Here $h_{\alpha_{\mathrm{L}}}(z)$ defined in (B.10) is equal to the zero mode of spin $\frac{1}{2}$ left-moving fermion with spin structure $\alpha_{\mathrm{L}}$. The explicit form of $S$ and its relation to $e^{-\Gamma\left[\sigma ; g_{z z}^{\mathrm{A}}\right]}$ can be found in [13]. In the manipulations in (3.10), we have used (2.24) and the following identities:

$$
\begin{aligned}
E\left(Z_{2}, Z_{1}\right) & =\frac{\vartheta\left[\alpha_{\mathrm{L}}\right]\left(\int_{Z_{1}}^{Z_{2}} \omega\right)}{h_{\alpha_{\mathrm{L}}}\left(Z_{1}\right) h_{\alpha_{\mathrm{L}}}\left(Z_{2}\right)}, \\
\frac{\prod_{r=3}^{N} E\left(Z_{2}, Z_{r}\right) \prod_{I} E\left(z_{I}, Z_{1}\right)}{\prod_{I} E\left(z_{I}, Z_{2}\right) \prod_{r=3}^{N} E\left(Z_{1}, Z_{r}\right)} \cdot \frac{\sigma^{2}\left(Z_{1}\right)}{\sigma^{2}\left(Z_{2}\right)} & =-\frac{\alpha_{1}}{\alpha_{2}} .
\end{aligned}
$$

(3.11) is proved by observing

$$
|\partial \rho(z)|^{2}=C|\sigma(z)|^{4}\left|\frac{\prod_{I} E\left(z, z_{I}\right)}{\prod_{r} E\left(z, Z_{r}\right)}\right|^{2},
$$

where $C$ is a quantity independent of $z$. From this expression we can derive

$$
\begin{aligned}
\frac{\alpha_{1}}{\alpha_{2}} & =\lim _{z \rightarrow Z_{1}, w \rightarrow Z_{2}} \frac{\left(z-Z_{1}\right) \partial \rho(z)}{\left(w-Z_{2}\right) \partial \rho(w)} \\
& =\lim _{z \rightarrow Z_{1}, w \rightarrow Z_{2}} \frac{z-Z_{1}}{w-Z_{2}} \exp \left[\int_{w}^{z} d u \partial \ln |\partial \rho(u)|^{2}\right] \\
& =-\frac{\prod_{r=3}^{N} E\left(Z_{2}, Z_{r}\right) \prod_{I} E\left(z_{I}, Z_{1}\right)}{\prod_{I} E\left(z_{I}, Z_{2}\right) \prod_{r=3}^{N} E\left(Z_{1}, Z_{r}\right)} \cdot \frac{\sigma^{2}\left(Z_{1}\right)}{\sigma^{2}\left(Z_{2}\right)}
\end{aligned}
$$

Combining eqs. (2.20), (3.9) and (3.10), it is straightforward to show that (3.7) is proportional to (3.1).

\subsection{Dimensional regularization}

The amplitudes given by the integral (2.5) with the integrand of the form (3.2) is not well-defined, because of the contact term divergences. In order to make them well-defined, we employ the dimensional regularization illustrated in subsection 2.2. The amplitudes are given in the form (2.5) with the integrand (2.26) with $d=10-8 Q^{2}$. The light-cone gauge amplitudes are finite for $Q^{2}>10$. As in the case of even spin structure, we can 
define the amplitudes as analytic functions of $Q^{2}$ and take the limit $Q \rightarrow 0$ to obtain those in $d=10$. In order to study the limit, we recast the light-cone gauge expression into a conformal gauge one. The noncritical version of (3.2) is given as

$$
\begin{aligned}
& \int\left[d X^{\mu} d \psi^{\mu} d \bar{\psi}^{\mu} d b d \bar{b} d c d \bar{c} d \beta d \bar{\beta} d \gamma d \bar{\gamma}\right]_{g_{z \bar{z}}^{\mathrm{A}}} e^{-S^{\mathrm{tot}}} \\
& \quad \times \prod_{K=1}^{6 g-6+2 N}\left[\oint_{C_{K}} \frac{d z}{\partial \rho} b_{z z}+\varepsilon_{K} \oint_{\bar{C}_{K}} \frac{d \overline{\bar{z}}}{\bar{\partial} \bar{\rho}} b_{\bar{z} \bar{z}}\right] \prod_{I}\left[X\left(z_{I}\right) \bar{X}\left(\bar{z}_{I}\right)\right] \\
& \quad \times \prod_{r} e^{-\frac{i Q^{2}}{\alpha_{r}} \mathcal{X}^{+}}\left(\hat{\tilde{\mathbf{z}}}_{I}^{(r)}, \hat{\tilde{\mathbf{z}}}_{I^{(r)}}\right) \\
& \quad \times V_{1}^{(-2,-1)}\left(Z_{1}, \bar{Z}_{1}\right) V_{2}^{(0,-1)}\left(Z_{2}, \bar{Z}_{2}\right) \prod_{r=3}^{N}\left[V_{r}^{(-1,-1)}\left(Z_{r}, \bar{Z}_{r}\right)\right] .
\end{aligned}
$$

Here the worldsheet theory of the longitudinal variables are taken to be the supersymmetric $X^{ \pm}$CFT. We discuss the correlation functions of the supersymmetric $X^{ \pm}$CFT for odd spin structures in appendix C.1. As is shown in appendix C.2, this expression is equal to

$$
\begin{aligned}
& \int\left[d X^{\mu} d \psi^{\mu} d \bar{\psi}^{\mu} d b d \bar{b} d c d \bar{c} d \beta d \bar{\beta} d \gamma d \bar{\gamma}\right]_{g_{z \bar{z}}^{\mathrm{A}}} e^{-S^{\mathrm{tot}}} \\
& \quad \times \prod_{K=1}^{6 g-6+2 N}\left[\oint_{C_{K}} \frac{d z}{\partial \rho} b_{z z}+\varepsilon_{K} \oint_{\bar{C}_{K}} \frac{d \bar{z}}{\bar{\partial} \bar{\rho}} b_{\bar{z} \bar{z}}\right] \prod_{I}\left[e^{\phi} T_{F}^{\mathrm{LC}}\left(z_{I}\right) e^{\bar{\phi}} \bar{T}_{F}^{\mathrm{LC}}\left(\bar{z}_{I}\right)\right] \\
& \quad \times \prod_{r} e^{-\frac{i Q^{2}}{\alpha_{r}} \mathcal{X}^{+}}\left(\hat{\tilde{\mathbf{z}}}_{I^{(r)}}, \hat{\tilde{\mathbf{z}}}_{I^{(r)}}\right) \\
& \quad \times V_{1}^{(-2,-1)}\left(Z_{1}, \bar{Z}_{1}\right) V_{2}^{(0,-1)}\left(Z_{2}, \bar{Z}_{2}\right) \prod_{r=3}^{N}\left[V_{r}^{(-1,-1)}\left(Z_{r}, \bar{Z}_{r}\right)\right] .
\end{aligned}
$$

In (3.15), we can replace $V_{2}^{(0,-1)}\left(Z_{2}, \bar{Z}_{2}\right)$ by (3.8) for the same reason as that in the critical case. With the replacement, the path integral (3.15) can factorize into those of matter, ghosts and superghosts. For the longitudinal variables, we get from (C.7)

$$
\begin{aligned}
\int\left[d \mathcal{X}^{+} d \mathcal{X}^{-}\right]_{g_{z \bar{z}}^{\mathrm{A}}} e^{-S_{\text {super }}^{ \pm}\left[g_{z \bar{z}}^{\mathrm{A}}\right]} \prod_{r=1}^{N}\left[V_{r}^{\mathrm{DDF}}\left(Z_{r}, \bar{Z}_{r}\right) e^{-\frac{i Q^{2}}{\alpha_{r}} X^{+}}\left(\hat{\tilde{\mathbf{z}}}_{I^{(r)}}, \hat{\tilde{\mathbf{z}}}_{I^{(r)}}\right)\right] \psi^{+}\left(Z_{1}\right) \psi^{-}\left(Z_{2}\right) \\
=(2 \pi)^{2} \delta\left(\sum_{s} p_{s}^{-}\right) \delta\left(\sum_{r} p_{r}^{+}\right) Z_{X^{ \pm}}\left[g_{z \bar{z}}^{\mathrm{A}}\right]\left(\frac{\operatorname{det}^{\prime}\left(-g^{\mathrm{A} z \bar{z}} \partial_{z} \partial_{\bar{z}}\right)}{\operatorname{det} \operatorname{Im} \Omega \int d^{2} z \sqrt{g^{\mathrm{A}}}}\right)^{-\frac{1}{2}} \vartheta\left[\alpha_{\mathrm{R}}\right](0)^{*} \\
\quad \times e^{\frac{Q^{2}}{2} \Gamma\left[\sigma ; g_{z \bar{z}}^{\mathrm{A}}\right]} h_{\alpha_{\mathrm{L}}}\left(Z_{1}\right) h_{\alpha_{\mathrm{L}}}\left(Z_{2}\right) \prod_{r}\left[\frac{1}{\alpha_{r}} e^{-\operatorname{Re} \bar{N}_{00}^{r r}} V_{r}^{\mathrm{LC}}\right]
\end{aligned}
$$

Combining eqs. (2.20), (3.10) and (3.16), it is straightforward to show that (3.15) is proportional to the light-cone gauge expression in (2.26) with $d=10-8 Q^{2}$.

The expression (3.14), summed over spin structures and integrated over the moduli parameters, gives a BRST invariant expression of the amplitude. The operators $X, \bar{X}$, $e^{-\frac{i Q^{2}}{\alpha_{r}} \mathcal{X}^{+}}\left(\hat{\tilde{\mathbf{z}}}_{I^{(r)}}, \hat{\tilde{\mathbf{z}}}_{I^{(r)}}\right), V_{1}^{(-2,-1)}, V_{2}^{(0,-1)}, V_{r}^{(-1,-1)}$ are all BRST invariant and the BRST 
variations of the antighost insertions yield total derivatives on moduli space. Since (3.14) coincides with (2.26), the amplitude is finite for $Q^{2}>10$.

We use the light-cone gauge expression of the amplitude for $Q^{2}>10$ to define it as an analytic function of $Q^{2}$, which is denoted by $A^{\mathrm{LC}}\left(Q^{2}\right)$. We would like to see what happens in the limit $Q \rightarrow 0$. The conformal gauge expression (3.14) can be deformed to define the amplitudes following the Sen-Witten prescription [23, 24]. We can divide the moduli space into patches and put the PCO's avoiding the spurious singularities as was explained in [23] and define the amplitude $A^{\mathrm{SW}}\left(Q^{2}\right)$. Moving the locations of the PCO's, the amplitudes change by total derivative terms in moduli space. Taking $Q^{2}$ big enough, these total derivative terms do not contribute to the amplitudes, because the infrared divergences are regularized. Therefore $A^{\mathrm{SW}}\left(Q^{2}\right)$ coincides with $A^{\mathrm{LC}}\left(Q^{2}\right)$ as an analytic function of $Q^{2}$. Since $A^{\mathrm{SW}}\left(Q^{2}\right)$ is free from the spurious singularities, it can be well-defined for $Q^{2}<10$ and

$$
\lim _{Q \rightarrow 0} A^{\mathrm{LC}}\left(Q^{2}\right)=A^{\mathrm{SW}}(0),
$$

if the right hand side is well-defined.

\section{Conclusions and discussions}

In this paper, we have shown that the Feynman amplitudes of the light-cone gauge closed superstring field theory can be calculated using the dimensional regularization technique, for higher genus Riemann surfaces with odd spin structure, if the external lines are in the (NS,NS) sector. In order to deal with the fermion zero modes peculiar to odd spin structures, we need to change the pictures of the vertex operators in the conformal gauge expression. We obtain the amplitudes in noncritical dimensions which coincide with the ones defined by using the Sen-Witten prescription. The amplitudes in the critical dimensions correspond to the limit $d \rightarrow 10$ or $Q \rightarrow 0$, and the results coincide with those given by the Sen-Witten prescription.

There are several things remain to be done. One is to check how the amplitudes obtained by our procedure are related to the standard results in more detail. In particular, we should study the conditionally convergent integrals which appear in the Feynman amplitudes of superstrings. We expect that our regularization makes the integrals well-defined but in a way different from those in $[25,26]$. Another thing to be done is to generalize our results to the amplitudes with external lines in the Ramond sector. With the correlation functions involving spin fields given for example in [27], it will be straightforward to rewrite the light-cone gauge expression into the conformal gauge one. These problems are left to future work.

\section{Acknowledgments}

N.I. would like to thank Ashoke Sen for useful comments. He would also like to thank the organizers of "Recent Developments on Light Front" at Arnold Sommerfeld Center for Theoretical Physics and "SFT@HIT" at Holon, especially Ivo Sachs, Ted Erler, Sebastian Konopka and Michael Kroyter, for hospitality. This work was supported in part by Grantin-Aid for Scientific Research (C) (25400242) and (15K05063) from MEXT. 


\section{A Operator valued coordinate}

It is convenient to introduce the operator valued coordinate $\hat{z}_{I}$ and its supersymmetric version $\hat{\tilde{\mathbf{z}}}_{I}$, in order to express the conformal gauge form of the amplitudes for noncritical dimensions.

Let us first consider $\hat{z}_{I}$ which is defined in the bosonic case. In the light-cone gauge setup, we consider the situation where the variable $X^{+}(z, \bar{z})$ possesses an expectation value $-\frac{i}{2}(\rho(z)+\bar{\rho}(\bar{z}))$. Therefore we decompose it into the expectation value and the fluctuation as

$$
X^{+}(z, \bar{z})=-\frac{i}{2}(\rho(z)+\bar{\rho}(\bar{z}))+\delta X^{+}(z, \bar{z}) .
$$

Roughly speaking, we define $\hat{z}_{I}$ to be an operator valued coordinate which satisfies

$$
\partial X^{+}\left(\hat{z}_{I}\right)=0 \text {. }
$$

Substituting (A.1) into (A.2), we get

$$
-\frac{i}{2} \partial \rho\left(\hat{z}_{I}\right)+\partial \delta X^{+}\left(\hat{z}_{I}\right)=0 .
$$

We take $\hat{z}_{I}$ so as to coincide with $z_{I}$ when $\delta X^{+}=0$. Assuming that $\hat{z}_{I}$ is expanded in terms of the fluctuation $\delta X^{+}$as

$$
\hat{z}_{I}=z_{I}+\sum_{n=1}^{\infty} \delta^{(n)} \hat{z}_{I}
$$

where $\delta^{(n)} z_{I}$ is at the $n$-th order in the derivatives of $\delta X^{+}$, in principle we can obtain $\delta^{(n)} z_{I}$ if $\partial^{2} \rho\left(z_{I}\right) \neq 0$. Lower order examples are given by

$$
\begin{aligned}
& \delta^{(1)} \hat{z}_{I}=-\frac{2 i}{\partial^{2} \rho} \partial \delta X^{+}\left(z_{I}\right) \\
& \delta^{(2)} \hat{z}_{I}=-\frac{4}{\left(\partial^{2} \rho\right)^{2}} \partial \delta X^{+} \partial^{2} \delta X^{+}\left(z_{I}\right)+\frac{2 \partial^{3} \rho}{\left(\partial^{2} \rho\right)^{3}}\left(\partial \delta X^{+}\right)^{2}\left(z_{I}\right) .
\end{aligned}
$$

In general $\delta^{(n)} z_{I}$ becomes a polynomial of the derivatives of $\delta X^{+}$at $z=z_{I}$. Quantities of order $n$ with $n>N$ for some $N>0$ do not contribute to the correlation functions we consider in this paper. $\hat{\bar{z}}_{I}$, which is the antiholomorphic counterpart of $\hat{z}_{I}$, can be obtained in the same way.

The OPE of $\hat{z}_{I}$ with the energy-momentum tensor $T(z)$ comes from the contractions of $\partial X^{-}$in $T(z)$ with $\partial^{k} \delta X^{+}\left(z_{I}\right)$ in $\hat{z}_{I}$. Taking the OPE of (A.2) with $T(z)$, we get

$$
\overparen{T(z) \hat{z}_{I}} \partial^{2} X^{+}\left(\hat{z}_{I}\right)+\frac{1}{\left(z-\hat{z}_{I}\right)^{2}} \partial X^{+}\left(\hat{z}_{I}\right)+\frac{1}{z-\hat{z}_{I}} \partial^{2} X^{+}\left(\hat{z}_{I}\right)=0 .
$$

Using (A.2) and the fact that $\partial^{2} X^{+}\left(\hat{z}_{I}\right)=-\frac{i}{2} \partial^{2} \rho\left(z_{I}\right)+\cdots$ is invertible perturbatively, we obtain

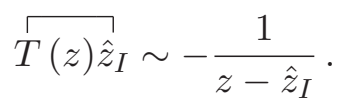


Expanding $\hat{z}_{I}$ as in (A.4), we can see that the right hand side of (A.7) involves poles of arbitrarily high order at $z=z_{I}$. Only finite number of them are relevant in the correlation functions (2.29), (3.14) and (3.15).

With the OPE (A.7) and its antiholomorphic version, we can show the OPE's

$$
\begin{aligned}
T(z) e^{i \alpha X^{+}}\left(\hat{z}_{I}, \hat{\bar{z}}_{I}\right) \sim \text { regular }, \\
\bar{T}(\bar{z}) e^{i \alpha X^{+}}\left(\hat{z}_{I}, \hat{\bar{z}}_{I}\right) \sim \text { regular },
\end{aligned}
$$

for any constant $\alpha$. Therefore $e^{i \alpha X^{+}}\left(\hat{z}_{I}, \hat{\bar{z}}_{I}\right)$ is a BRST invariant operator in the conformal gauge bosonic string theory in noncritical dimensions.

It is straightforward to define the operator valued supercoordinate $\hat{\tilde{\mathbf{z}}}_{I}$. We define $\hat{\tilde{\mathbf{z}}}_{I}=\left(\hat{\tilde{z}}_{I}, \hat{\tilde{\theta}}_{I}\right)$ to be the operator valued supercoordinate which satisfies

$$
\begin{aligned}
\partial \mathcal{X}^{+}\left(\hat{\tilde{\mathbf{z}}}_{I}\right) & =0, \\
\partial D \mathcal{X}^{+}\left(\hat{\tilde{\mathbf{z}}}_{I}\right) & =0 .
\end{aligned}
$$

Notice that $\hat{\tilde{\mathbf{z}}}_{I}$ is the operator version of the supercoordinate $\tilde{\mathbf{z}}_{I}$ defined in $[7,28,29]$ which is not superconformal, but it is sufficient for our purpose. Similarly to the bosonic case, we decompose $\mathcal{X}^{+}(\mathbf{z}, \overline{\mathbf{z}})$ as

$$
\mathcal{X}^{+}(\mathbf{z}, \overline{\mathbf{z}})=-\frac{i}{2}\left(\rho_{s}(\mathbf{z})+\bar{\rho}_{s}(\overline{\mathbf{z}})\right)+\delta \mathcal{X}^{+}(\mathbf{z}, \overline{\mathbf{z}})
$$

where $\rho_{s}, \bar{\rho}_{s}$ are the supersymmetric version of $\rho, \bar{\rho}$ whose explicit form is given in (C.5). $S_{\alpha}$ in that equation is the one in (B.5) or in (B.12) according to whether the spin structure of the fermions is even or odd. Using this decomposition, we get $\hat{\tilde{z}}_{I}, \hat{\tilde{\theta}}_{I}$ as expansions around $\tilde{z_{I}}, \tilde{\theta}_{I}$ in terms of the fluctuation $\delta \mathcal{X}^{+}$as

$$
\begin{aligned}
& \hat{\tilde{z}}_{I}=\tilde{z}_{I}+\sum_{n=1}^{\infty} \delta^{(n)} \hat{\tilde{z}}_{I}, \\
& \hat{\tilde{\theta}}_{I}=\tilde{\theta}_{I}+\sum_{n=1}^{\infty} \delta^{(n)} \hat{\tilde{\theta}}_{I},
\end{aligned}
$$

assuming $\partial^{2} \rho\left(\tilde{\mathbf{z}}_{I}\right) \neq 0$. For example,

$$
\begin{aligned}
& \delta^{(1)} \hat{\tilde{z}}_{I}=-\frac{2 i}{\partial^{2} \rho} \partial \delta \mathcal{X}^{+}\left(\tilde{\mathbf{z}}_{I}\right), \\
& \delta^{(1)} \hat{\tilde{\theta}}_{I}=-\frac{2 i}{\partial^{2} \rho} \partial D \delta \mathcal{X}^{+}\left(\tilde{\mathbf{z}}_{I}\right)
\end{aligned}
$$


We obtain the OPE's

$$
\begin{aligned}
& \prod_{T(\mathbf{z}) \hat{\tilde{z}}_{I}}-T(\mathbf{z}) \hat{\tilde{\theta}}_{I} \hat{\tilde{\theta}}_{I} \sim-\frac{\theta-\hat{\tilde{\theta}}_{I}}{\mathbf{z}-\hat{\tilde{\mathbf{z}}}_{I}}-\frac{1}{\left(\mathbf{z}-\hat{\tilde{\mathbf{z}}}_{I}\right)^{2}} \cdot \frac{D \mathcal{X}^{+}}{2 \partial^{2} \mathcal{X}^{+}}\left(\hat{\tilde{\mathbf{z}}}_{I}\right), \\
& T(\mathbf{z}) \hat{\tilde{\theta}}_{I} \sim-\frac{\frac{1}{2}}{\mathbf{z}-\hat{\tilde{\mathbf{z}}}_{I}}-\frac{\theta-\hat{\tilde{\theta}}_{I}}{\left(\mathbf{z}-\hat{\tilde{\mathbf{z}}}_{I}\right)^{3}} \cdot \frac{D \mathcal{X}^{+}}{\partial^{2} \mathcal{X}^{+}}\left(\hat{\tilde{\mathbf{z}}}_{I}\right) \\
& -\frac{1}{\left(\mathbf{z}-\hat{\tilde{\mathbf{z}}}_{I}\right)^{2}} \cdot \frac{\partial^{2} D \mathcal{X}^{+} D \mathcal{X}^{+}}{2\left(\partial^{2} \mathcal{X}^{+}\right)^{2}}\left(\hat{\tilde{\mathbf{z}}}_{I}\right) \text {. }
\end{aligned}
$$

From these OPE's, we get

$$
\begin{aligned}
& T(\mathbf{z}) e^{i \alpha \mathcal{X}^{+}}\left(\hat{\tilde{\mathbf{z}}}_{I}, \hat{\tilde{\mathbf{z}}}_{I}\right) \sim \text { regular }, \\
& \bar{T}(\overline{\mathbf{z}}) e^{i \alpha \mathcal{X}^{+}}\left(\hat{\tilde{\mathbf{z}}}_{I}, \hat{\tilde{\mathbf{z}}}_{I}\right) \sim \text { regular },
\end{aligned}
$$

for any constant $\alpha$. Therefore $e^{i \alpha \mathcal{X}^{+}}\left(\hat{\tilde{\mathbf{z}}}_{I}, \hat{\tilde{\mathbf{z}}}_{I}\right)$ is BRST invariant in the conformal gauge superstring theory in noncritical dimensions.

Using the operator valued coordinate thus defined, we can define a BRST invariant operator

$$
e^{-\frac{i Q^{2}}{\alpha_{r}} \mathcal{X}^{+}}\left(\hat{\tilde{\mathbf{z}}}_{I^{(r)}}, \hat{\tilde{\mathbf{z}}}_{I^{(r)}}\right)
$$

which can be replaced by

$$
e^{-\frac{i Q^{2}}{\alpha_{r}} X^{+}}\left(z_{I^{(r)}}, \bar{z}_{I^{(r)}}\right)
$$

in evaluating (2.29), (3.14) and (3.15). This expression can be used instead of the complicated combination $\oint_{z_{I}^{(r)}} \frac{d z}{2 \pi i} \oint_{\bar{z}_{I}^{(r)}} \frac{d \bar{z}}{2 \pi i} S\left(z, Z_{r}\right) \bar{S}\left(\bar{z}, \bar{Z}_{r}\right)$ in $[13,30]$, which has a similar effect.

\section{B Correlation functions of free fermions}

In this appendix, we review a few basic facts about the correlation functions of free fermions on higher genus Riemann surfaces.

The correlation functions of a free Dirac fermion with spin structure $\alpha_{\mathrm{L}}$ for left and $\alpha_{\mathrm{R}}$ for right can be given by $[19,31]$

$$
\begin{gathered}
\int\left[d \psi d \bar{\psi} d \bar{\psi}^{\dagger} d \psi^{\dagger}\right]_{\hat{g}_{z \bar{z}}} e^{-S} \psi^{\dagger}\left(x_{1}\right) \bar{\psi}^{\dagger}\left(\bar{x}_{1}\right) \cdots \psi^{\dagger}\left(x_{n}\right) \bar{\psi}^{\dagger}\left(\bar{x}_{n}\right) \bar{\psi}\left(\bar{y}_{n}\right) \psi\left(y_{n}\right) \cdots \bar{\psi}\left(\bar{y}_{1}\right) \psi\left(y_{1}\right) \\
=\left(\frac{\operatorname{det}^{\prime}\left(-\hat{g}^{z \bar{z}} \partial_{z} \partial_{\bar{z}}\right)}{\operatorname{det} \operatorname{Im} \Omega \int d^{2} z \sqrt{\hat{g}}}\right)^{-\frac{1}{2}} \\
\quad \times \vartheta\left[\alpha_{\mathrm{L}}\right]\left(\sum_{i=1}^{n} \int_{P_{0}}^{x_{i}} \omega-\sum_{i=1}^{n} \int_{P_{0}}^{y_{i}} \omega\right) \vartheta\left[\alpha_{\mathrm{R}}\right]\left(\sum_{i=1}^{n} \int_{P_{0}}^{x_{i}} \omega-\sum_{i=1}^{n} \int_{P_{0}}^{y_{i}} \omega\right)^{*} \\
\quad \times\left|\frac{\prod_{i<j}\left[E\left(x_{i}, x_{j}\right) E\left(y_{j}, y_{i}\right)\right]}{\prod_{i, j} E\left(x_{i}, y_{j}\right)}\right|^{2}
\end{gathered}
$$


When both $\alpha_{\mathrm{L}}$ and $\alpha_{\mathrm{R}}$ correspond to even spin structures, using the formula

$$
\begin{aligned}
& \vartheta\left(\sum_{i=1}^{n} \int_{P_{0}}^{x_{i}} \omega-\sum_{i=1}^{n} \int_{P_{0}}^{y_{i}} \omega-e\right) \vartheta(e)^{n-1} \frac{\prod_{i<j}\left[E\left(x_{i}, x_{j}\right) E\left(y_{j}, y_{i}\right)\right]}{\prod_{i, j} E\left(x_{i}, y_{j}\right)} \\
& =\operatorname{det}\left(\frac{\vartheta\left(\int_{P_{0}}^{x_{i}} \omega-\int_{P_{0}}^{y_{i}} \omega-e\right)}{E\left(x_{i}, y_{j}\right)}\right),
\end{aligned}
$$

given in [32] for the case

$$
e_{\nu}=-\left(\Omega \alpha^{\prime}+\alpha^{\prime \prime}\right)_{\nu},
$$

it is straightforward to show that (B.1) can be transformed into

$$
\begin{aligned}
& \int\left[d \psi d \bar{\psi} d \bar{\psi}^{\dagger} d \psi^{\dagger}\right]_{\hat{g}_{z \bar{z}}} e^{-S} \psi^{\dagger}\left(x_{1}\right) \bar{\psi}^{\dagger}\left(\bar{x}_{1}\right) \cdots \psi^{\dagger}\left(x_{n}\right) \bar{\psi}^{\dagger}\left(\bar{x}_{n}\right) \bar{\psi}\left(\bar{y}_{n}\right) \psi\left(y_{n}\right) \cdots \bar{\psi}\left(\bar{y}_{1}\right) \psi\left(y_{1}\right) \\
& =\left(\frac{\operatorname{det}^{\prime}\left(-\hat{g}^{z \bar{z}} \partial_{z} \partial_{\bar{z}}\right)}{\operatorname{det} \operatorname{Im} \Omega \int d^{2} z \sqrt{\hat{g}}}\right)^{-\frac{1}{2}} \vartheta\left[\alpha_{\mathrm{L}}\right](0) \vartheta\left[\alpha_{\mathrm{R}}\right](0)^{*} \operatorname{det}\left[S_{\alpha_{\mathrm{L}}}\left(x_{i}, y_{j}\right)\right] \operatorname{det}\left[S_{\alpha_{\mathrm{R}}}\left(x_{i}, y_{j}\right)^{*}\right]
\end{aligned}
$$

where

$$
S_{\alpha}(z, w)=\frac{1}{E(z, w)} \frac{\vartheta[\alpha]\left(\int_{w}^{z} \omega\right)}{\vartheta[\alpha](0)}
$$

is the Szego kernel. The expression (B.4) implies that the partition function is given by

$$
\left(Z^{\psi}\left[g_{z \bar{z}}\right]\right)^{2}=\left(\frac{\operatorname{det}^{\prime}\left(-g^{z \bar{z}} \partial_{z} \partial_{\bar{z}}\right)}{\operatorname{det} \operatorname{Im} \Omega \int d^{2} z \sqrt{g}}\right)^{-\frac{1}{2}} \vartheta\left[\alpha_{\mathrm{L}}\right](0) \vartheta\left[\alpha_{\mathrm{R}}\right](0)^{*}
$$

and the propagators of the fermions are

$$
\begin{aligned}
\overparen{\psi^{\dagger}(x)} \psi(y) & =S_{\alpha_{\mathrm{L}}}(x, y), \\
\overline{\psi^{\dagger}(\bar{x}) \bar{\psi}}(\bar{y}) & =S_{\alpha_{\mathrm{R}}}(x, y)^{*}
\end{aligned}
$$

When the spin structures are not even, we need to take care of the fermion zero modes. For example, let us consider the case where $\alpha_{\mathrm{L}}$ corresponds to an odd spin structure and $\alpha_{\mathrm{R}}$ corresponds to an even one. In this case, using the formula (B.2) for

$$
e_{\nu}=-\left(\Omega \alpha_{\mathrm{L}}^{\prime}+\alpha_{\mathrm{L}}^{\prime \prime}\right)_{\nu}-\int_{q}^{p} \omega_{\nu}
$$

in the limit $q \rightarrow p$, we get [32]

$$
\begin{aligned}
& \vartheta\left[\alpha_{\mathrm{L}}\right]\left(\sum_{i=1}^{n} \int_{P_{0}}^{x_{i}} \omega-\sum_{i=1}^{n} \int_{P_{0}}^{y_{i}} \omega\right) \frac{\prod_{i<j}\left[E\left(x_{i}, x_{j}\right) E\left(y_{j}, y_{i}\right)\right]}{\prod_{i, j} E\left(x_{i}, y_{j}\right)} \\
& =\int d \psi_{0} d \psi_{0}^{\dagger} \operatorname{det}\left[\psi_{0}^{\dagger} h_{\alpha_{\mathrm{L}}}\left(x_{i}\right) \psi_{0} h_{\alpha_{\mathrm{L}}}\left(y_{j}\right)+\frac{1}{E\left(x_{i}, y_{j}\right)} \frac{\sum_{\nu} \partial_{\nu} \vartheta\left[\alpha_{\mathrm{L}}\right]\left(\int_{y_{j}}^{x_{i}} \omega\right) \omega_{\nu}(p)}{\sum_{\nu} \partial_{\nu} \vartheta\left[\alpha_{\mathrm{L}}\right](0) \omega_{\nu}(p)}\right] .
\end{aligned}
$$


Here

$$
h_{\alpha_{\mathrm{L}}}(z)=\sqrt{\sum_{\nu} \partial_{\nu} \vartheta\left[\alpha_{\mathrm{L}}\right](0) \omega_{\nu}(z)}
$$

gives the zero mode of the fermion. Substituting (B.9) into the right hand side of (B.4), we obtain

$$
\begin{gathered}
\int\left[d \psi d \bar{\psi} d \bar{\psi}^{\dagger} d \psi^{\dagger}\right]_{\hat{g}_{z \bar{z}}} e^{-S} \psi^{\dagger}\left(x_{1}\right) \bar{\psi}^{\dagger}\left(\bar{x}_{1}\right) \cdots \psi^{\dagger}\left(x_{n}\right) \bar{\psi}^{\dagger}\left(\bar{x}_{n}\right) \bar{\psi}\left(\bar{y}_{n}\right) \psi\left(y_{n}\right) \cdots \bar{\psi}\left(\bar{y}_{1}\right) \psi\left(y_{1}\right) \\
=\left(\frac{\operatorname{det}^{\prime}\left(-\hat{g}^{z \bar{z}} \partial_{z} \partial_{\bar{z}}\right)}{\operatorname{det} \operatorname{Im} \Omega \int d^{2} z \sqrt{\hat{g}}}\right)^{-\frac{1}{2}} \int d \psi_{0} d \psi_{0}^{\dagger} \operatorname{det}\left[S_{\alpha_{\mathrm{L}}}\left(x_{i}, y_{j}\right)\right] \vartheta\left[\alpha_{\mathrm{R}}\right](0)^{*} \operatorname{det}\left[S_{\alpha_{\mathrm{R}}}\left(x_{i}, y_{j}\right)^{*}\right],
\end{gathered}
$$

where

$$
S_{\alpha_{\mathrm{L}}}(x, y)=\psi_{0}^{\dagger} h_{\alpha_{\mathrm{L}}}(x) \psi_{0} h_{\alpha_{\mathrm{L}}}(y)+\frac{1}{E(x, y)} \frac{\sum_{\nu} \partial_{\nu} \vartheta\left[\alpha_{\mathrm{L}}\right]\left(\int_{y}^{x} \omega\right) \omega_{\nu}(p)}{\sum_{\nu} \partial_{\nu} \vartheta\left[\alpha_{\mathrm{L}}\right](0) \omega_{\nu}(p)}
$$

$S_{\alpha_{\mathrm{L}}}(x, y)$ can be identified with the propagator of the left-moving fermions and it involves the zero mode variables $\psi_{0}^{\dagger}, \psi_{0} \cdot \psi_{0}^{\dagger}$ and $\psi_{0}$ should be integrated over after all the contractions are performed. The other cases where $\alpha_{\mathrm{R}}$ corresponds to an odd spin structure can be dealt with in the same way.

\section{Dimensional regularization for odd spin structure}

In this appendix, we explain the details of how dimensional regularization works in the case of odd spin structure.

\section{C.1 Supersymmetric $X^{ \pm}$CFT}

In order to get the expression of the amplitudes in the conformal gauge, we need to calculate the correlation functions of the supersymmetric $X^{ \pm}$CFT on the surface with odd spin structure.

The action of the supersymmetric $X^{ \pm}$CFT is given in the form

$$
S_{\text {super }}^{ \pm}\left[\hat{g}_{z \bar{z}}, \mathcal{X}^{ \pm}\right]=S_{\text {free }}\left[\hat{g}_{z \bar{z}}, \mathcal{X}^{ \pm}\right]+\frac{d-10}{8} \Gamma_{\text {super }}\left[\hat{g}_{z \bar{z}}, 2 i \mathcal{X}^{+}\right]
$$

where $S_{\text {free }}\left[\hat{g}_{z \bar{z}}, \mathcal{X}^{ \pm}\right]$denotes the free action of $\mathcal{X}^{ \pm}$. When the spin structures are both 
even, the correlation functions of the supersymmetric $X^{ \pm}$CFT are evaluated as [13]

$$
\begin{gathered}
\int\left[d \mathcal{X}^{+} d \mathcal{X}^{-}\right]_{\hat{g}_{z z}} e^{-S_{\text {super }}^{ \pm}\left[\hat{g}_{z \bar{z}}, \mathcal{X}^{ \pm}\right]} \prod_{r=1}^{N} e^{-i p_{r}^{+} \mathcal{X}^{-}}\left(\mathbf{Z}_{r}, \overline{\mathbf{Z}}_{r}\right) \prod_{u=1}^{M} e^{-i p_{u}^{-} \mathcal{X}^{+}}\left(\mathbf{w}_{u}, \overline{\mathbf{w}}_{u}\right) \\
=\int\left[d \mathcal{X}^{+} d \mathcal{X}^{-}\right]_{\hat{g}_{z \bar{z}}} e^{-S_{\text {free }}\left[\hat{g}_{z \bar{z}}, \mathcal{X}^{ \pm}\right]} \prod_{r=1}^{N} e^{-i p_{r}^{+} \mathcal{X}^{-}}\left(\mathbf{Z}_{r}, \overline{\mathbf{Z}}_{r}\right) \\
\times e^{-\frac{d-10}{8} \Gamma_{\text {super }}\left[\hat{g}_{z \bar{z}}, 2 i \mathcal{X}^{+}\right]} \prod_{u=1}^{M} e^{-i p_{u}^{-} \mathcal{X}^{+}}\left(\mathbf{w}_{u}, \overline{\mathbf{w}}_{u}\right) \\
=(2 \pi)^{2} \delta\left(\sum_{u} p_{u}^{-}\right) \delta\left(\sum_{r} p_{r}^{+}\right)\left(Z_{\text {super }}^{\mathcal{X}}\left[\hat{g}_{z \bar{z}}\right]\right)^{2} \\
\times e^{-\frac{d-10}{8} \Gamma_{\text {super }}\left[\hat{g}_{z \bar{z}}, \rho_{s}+\bar{\rho}_{s}\right]} \prod_{u} e^{-p_{u}^{-\frac{\rho_{s}+\bar{\rho}_{s}}{2}}}\left(\mathbf{w}_{u}, \overline{\mathbf{w}}_{u}\right)
\end{gathered}
$$

Regarding the second and third lines as a correlation function of

$$
e^{-\frac{d-10}{8} \Gamma_{\text {super }}\left[\hat{g}_{z \bar{z}}, 2 i \mathcal{X}^{+}\right]} \prod_{u=1}^{M} e^{-i p_{u}^{-} \mathcal{X}^{+}}\left(\mathbf{w}_{u}, \overline{\mathbf{w}}_{u}\right)
$$

of the free theory with the source term

$$
\prod_{r=1}^{N} e^{-i p_{r}^{+} \mathcal{X}^{-}}\left(\mathbf{Z}_{r}, \overline{\mathbf{Z}}_{r}\right)
$$

we can calculate it by replacing the $\mathcal{X}^{+}(\mathbf{z}, \overline{\mathbf{z}})$ by its expectation value $-\frac{i}{2}\left(\rho_{s}(\mathbf{z})+\bar{\rho}_{s}(\overline{\mathbf{z}})\right)$ and derive the fourth line. Here $\rho_{s}, \bar{\rho}_{s}$ are the supersymmetric version of $\rho, \bar{\rho}$ and expressed as

$$
\begin{aligned}
& \rho_{s}(\mathbf{z})=\rho(z)-\theta \sum_{r} \alpha_{r} \Theta_{r} S_{\alpha_{\mathrm{L}}}\left(z, Z_{r}\right), \\
& \bar{\rho}_{s}(\overline{\mathbf{z}})=\bar{\rho}(\bar{z})-\bar{\theta} \sum_{r} \alpha_{r} \bar{\Theta}_{r} S_{\alpha_{\mathrm{R}}}\left(\bar{z}, \bar{Z}_{r}\right),
\end{aligned}
$$

where $S_{\alpha_{\mathrm{L}}}$ and $S_{\alpha_{\mathrm{R}}}$ are taken to be the Szego kernel (B.5). The partition function $\left(Z_{\text {super }}^{\mathcal{X}}\left[\hat{g}_{z \bar{z}}\right]\right)^{2}$ is described by using $Z_{X^{ \pm}}\left[\hat{g}_{z \bar{z}}\right]$ and $Z_{\psi^{ \pm}}\left[\hat{g}_{z \bar{z}}\right]$ in $(2.19)$ as

$$
\left(Z_{\text {super }}^{\mathcal{X}}\left[\hat{g}_{z \bar{z}}\right]\right)^{2}=Z_{X^{ \pm}}\left[\hat{g}_{z \bar{z}}\right] Z_{\psi^{ \pm}}\left[\hat{g}_{z \bar{z}}\right]
$$

The explicit form of $e^{-\frac{d-10}{8} \Gamma_{\text {super }}\left[\hat{g}_{z \bar{z}}, \rho_{s}+\bar{\rho}_{s}\right]}$ can be found in [13].

In the case where $\alpha_{\mathrm{L}}$ corresponds to an odd spin structure, we can proceed in the same way. Since the correlation functions of the free fermions are given in (B.11) as an integral 
over the zero modes $\psi_{0}^{ \pm}$, we obtain

$$
\begin{gathered}
\int\left[d \mathcal{X}^{+} d \mathcal{X}^{-}\right]_{\hat{g}_{z \bar{z}}} e^{-S_{\text {super }}^{ \pm}\left[\hat{g}_{z \bar{z}}\right]} \prod_{r=1}^{N} e^{-i p_{r}^{+} \mathcal{X}^{-}}\left(\mathbf{Z}_{r}, \overline{\mathbf{Z}}_{r}\right) \prod_{u=1}^{M} e^{-i p_{u}^{-} \mathcal{X}^{+}}\left(\mathbf{w}_{u}, \overline{\mathbf{w}}_{u}\right) \\
=(2 \pi)^{2} \delta\left(\sum_{u} p_{u}^{-}\right) \delta\left(\sum_{r} p_{r}^{+}\right) Z_{X^{ \pm}}\left[\hat{g}_{z \bar{z}}\right]\left(\frac{\operatorname{det}^{\prime}\left(-\hat{g}^{z \bar{z}} \partial_{z} \partial_{\bar{z}}\right)}{\operatorname{det} \operatorname{Im} \Omega \int d^{2} z \sqrt{\hat{g}}}\right)^{-\frac{1}{2}} \vartheta\left[\alpha_{\mathrm{R}}\right](0)^{*} \\
\quad \times \int d \psi_{0}^{+} d \psi_{0}^{-} \prod_{u} e^{-p_{u}^{-\frac{\rho_{s}+\bar{\rho}_{s}}{2}}}\left(\mathbf{w}_{u}, \overline{\mathbf{w}}_{u}\right) e^{-\frac{d-10}{8} \Gamma_{\text {super }}\left[\hat{g}_{z \bar{z}}, \rho_{s}+\bar{\rho}_{s}\right]}
\end{gathered}
$$

where $\rho_{s}, \bar{\rho}_{s}$ in this formula are (C.5) with $S_{\alpha_{\mathrm{L}}}$ given in (B.12) and $S_{\alpha_{\mathrm{R}}}$ taken to be the Szego kernel (B.5).

With the correlation function (C.7), it is straightforward to check the following properties of the energy-momentum tensor:

- $T^{\mathcal{X}^{ \pm}}(\mathbf{z})$ is regular at $z=z_{I}$.

- $T^{\mathcal{X}^{ \pm}}(\mathbf{z})$ satisfies

$$
\begin{aligned}
T^{\mathcal{X}^{ \pm}}(\mathbf{z}) e^{-i p_{r}^{+} \mathcal{X}^{-}-i p_{r}^{-} \mathcal{X}^{+}}\left(\mathbf{Z}_{r}, \overline{\mathbf{Z}}_{r}\right) \sim & \frac{\theta-\Theta_{r}}{\left(z-Z_{r}\right)^{2}}\left(-p_{r}^{+} p_{r}^{-}\right) e^{-i p_{r}^{+} \mathcal{X}^{-}-i p_{r}^{-} \mathcal{X}^{+}}\left(\mathbf{Z}_{r}, \overline{\mathbf{Z}}_{r}\right) \\
& +\frac{1}{\mathbf{Z}-\mathbf{Z}_{r}} \frac{1}{2} D e^{-i p_{r}^{+} \mathcal{X}^{-}-i p_{r}^{-} \mathcal{X}^{+}}\left(\mathbf{Z}_{r}, \overline{\mathbf{Z}}_{r}\right) \\
& +\frac{\theta-\Theta_{r}}{z-Z_{r}} \partial e^{-i p_{r}^{+} \mathcal{X}^{-}-i p_{r}^{-} \mathcal{X}^{+}}\left(\mathbf{Z}_{r}, \overline{\mathbf{Z}}_{r}\right)
\end{aligned}
$$

- The OPE between $\mathcal{X}^{-}$'s is given by

$$
\begin{aligned}
& D \mathcal{X}^{-}(\mathbf{z}) D \mathcal{X}^{-}\left(\mathbf{z}^{\prime}\right) \\
& \sim-\frac{d-10}{4} \\
& \times D D^{\prime}\left[\frac{\theta-\theta^{\prime}}{\left(\mathbf{z}-\mathbf{z}^{\prime}\right)^{3}} \frac{3 D \mathcal{X}^{+}}{\left(\partial \mathcal{X}^{+}\right)^{3}}\left(\mathbf{z}^{\prime}\right)\right. \\
& +\frac{1}{\left(\mathbf{z}-\mathbf{z}^{\prime}\right)^{2}}\left(\frac{1}{2\left(\partial \mathcal{X}^{+}\right)^{2}}+\frac{4 \partial D \mathcal{X}^{+} D \mathcal{X}^{+}}{\left(\partial \mathcal{X}^{+}\right)^{4}}\right)\left(\mathbf{z}^{\prime}\right) \\
& +\frac{\theta-\theta^{\prime}}{\left(\mathbf{z}-\mathbf{z}^{\prime}\right)^{2}}\left(-\frac{\partial D \mathcal{X}^{+}}{\left(\partial \mathcal{X}^{+}\right)^{3}}-\frac{5 \partial^{2} \mathcal{X}^{+} D \mathcal{X}^{+}}{2\left(\partial \mathcal{X}^{+}\right)^{4}}\right)\left(\mathbf{z}^{\prime}\right) \\
& +\frac{1}{\mathbf{z}-\mathbf{z}^{\prime}}\left(-\frac{\partial^{2} \mathcal{X}^{+}}{2\left(\partial \mathcal{X}^{+}\right)^{3}}+\frac{2 \partial^{2} D \mathcal{X}^{+} D \mathcal{X}^{+}}{\left(\partial \mathcal{X}^{+}\right)^{4}}-\frac{8 \partial^{2} \mathcal{X}^{+} \partial D \mathcal{X}^{+} D \mathcal{X}^{+}}{\left(\partial \mathcal{X}^{+}\right)^{5}}\right)\left(\mathbf{z}^{\prime}\right) \\
& +\frac{\theta-\theta^{\prime}}{\mathbf{z}-\mathbf{z}^{\prime}}\left(-\frac{\partial^{2} D \mathcal{X}^{+}}{2\left(\partial \mathcal{X}^{+}\right)^{3}}+\frac{3 \partial^{2} \mathcal{X}^{+} \partial D \mathcal{X}^{+}}{2\left(\partial \mathcal{X}^{+}\right)^{4}}-\frac{\partial^{3} \mathcal{X}^{+} D \mathcal{X}^{+}}{2\left(\partial \mathcal{X}^{+}\right)^{4}}\right. \\
& \left.\left.\quad+\frac{\left(\partial^{2} \mathcal{X}^{+}\right)^{2} D \mathcal{X}^{+}}{\left(\partial \mathcal{X}^{+}\right)^{5}}-\frac{\partial^{2} D \mathcal{X}^{+} \partial D \mathcal{X}^{+} D \mathcal{X}^{+}}{\left(\partial \mathcal{X}^{+}\right)^{5}}\right)\left(\mathbf{z}^{\prime}\right)\right], \quad(\mathrm{C} .9)
\end{aligned}
$$


and we can deduce that the energy momentum tensor $T^{\mathcal{X}^{ \pm}}(\mathbf{z})$ satisfies the OPE

$$
\begin{aligned}
& T^{\mathcal{X}^{ \pm}}(\mathbf{z}) T^{\mathcal{X}^{ \pm}}\left(\mathbf{z}^{\prime}\right) \\
& \quad \sim \frac{12-d}{4\left(\mathbf{z}-\mathbf{z}^{\prime}\right)^{3}}+\frac{\theta-\theta^{\prime}}{\left(\mathbf{z}-\mathbf{z}^{\prime}\right)^{2}} \frac{3}{2} T^{\mathcal{X}^{ \pm}}\left(\mathbf{z}^{\prime}\right)+\frac{1}{\mathbf{z}-\mathbf{z}^{\prime}} \frac{1}{2} D T^{\mathcal{X}^{ \pm}}\left(\mathbf{z}^{\prime}\right)+\frac{\theta-\theta^{\prime}}{\mathbf{z}-\mathbf{z}^{\prime}} \partial T^{\mathcal{X}^{ \pm}}\left(\mathbf{z}^{\prime}\right),
\end{aligned}
$$

which corresponds to the super Virasoro algebra with the central charge $\hat{c}=12-d$. It follows that combined with the transverse variables $X^{i}(\mathbf{z}, \overline{\mathbf{z}})$, the total central charge of the system becomes $\hat{c}=10$. This implies that with the ghost superfields $B(\mathbf{z})$ and $C(\mathbf{z})$ defined as

$$
B(\mathbf{z})=\beta(z)+\theta b(z), \quad C(\mathbf{z})=c(z)+\theta \gamma(z),
$$

it is possible to construct a nilpotent BRST charge

$$
Q_{\mathrm{B}}=\oint \frac{d \mathbf{z}}{2 \pi i}\left[-C\left(T^{\mathcal{X}^{ \pm}}-\frac{1}{2} D \mathcal{X}^{i} \partial \mathcal{X}^{i}\right)+\left(C \partial C-\frac{1}{4}(D C)^{2}\right) B\right] .
$$

These properties can be proved in the same way as in the even spin structure case, because we need only the behaviors of the fermion propagators around the singularities to do so.

In the same way as in the even spin structure case [13], we can derive from (C.7),

$$
\begin{aligned}
& \int\left[d \mathcal{X}^{+} d \mathcal{X}^{-}\right]_{\hat{g}_{z \bar{z}}} e^{-S_{\mathrm{super}}^{ \pm}\left[\hat{g}_{z \bar{z}}\right]} \prod_{r=1}^{N} e^{-i p_{r}^{+} X^{-}}\left(Z_{r}, \bar{Z}_{r}\right) \prod_{s=1}^{M} e^{-i p_{s}^{-} X^{+}}\left(w_{s}, \bar{w}_{s}\right) \\
& \times \psi^{+}\left(u_{1}\right) \cdots \psi^{+}\left(u_{n}\right) \psi^{-}\left(v_{1}\right) \cdots \psi^{-}\left(v_{m}\right) \\
& \times \bar{\psi}^{+}\left(\tilde{u}_{1}\right) \cdots \bar{\psi}^{+}\left(\tilde{u}_{n}\right) \bar{\psi}^{-}\left(\tilde{v}_{1}\right) \cdots \bar{\psi}^{-}\left(\tilde{v}_{m}\right) \\
&=(2 \pi)^{2} \delta\left(\sum_{s} p_{s}^{-}\right) \delta\left(\sum_{r} p_{r}^{+}\right) Z_{X^{ \pm}}\left[\hat{g}_{z \bar{z}}\right] \prod_{s} e^{-p_{s}^{-} \frac{1}{2}(\rho+\bar{\rho})}\left(w_{s}, \bar{w}_{s}\right) e^{-\frac{d-10}{16} \Gamma\left[\sigma ; \hat{g}_{z \bar{z}}\right]} \\
& \times \int\left[d \psi^{+} d \psi^{-} d \bar{\psi}^{+} d \bar{\psi}^{-}\right]_{\hat{g}_{z \bar{z}}} e^{\frac{1}{\pi} \int d^{2} z\left(\psi^{-} \bar{\partial} \psi^{+}+\bar{\psi}^{-} \partial \bar{\psi}^{+}\right)-S_{\mathrm{int}}} \\
& \times \psi^{+}\left(u_{1}\right) \cdots \psi^{+}\left(u_{n}\right) \psi^{-}\left(v_{1}\right) \cdots \psi^{-}\left(v_{m}\right) \\
& \times \bar{\psi}^{+}\left(\tilde{u}_{1}\right) \cdots \bar{\psi}^{+}\left(\tilde{u}_{n}\right) \bar{\psi}^{-}\left(\tilde{v}_{1}\right) \cdots \bar{\psi}^{-}\left(\tilde{v}_{m}\right)
\end{aligned}
$$

where

$$
\begin{aligned}
S_{\text {int }}=\frac{d-10}{8}[- & \sum_{r} \frac{2}{\alpha_{r}} \frac{\partial \psi^{+} \psi^{+}}{\partial^{2} \rho}\left(z_{I^{(r)}}\right) \\
& +\sum_{I}\left\{\left(\frac{5}{3} \frac{\partial^{4} \rho}{\left(\partial^{2} \rho\right)^{3}}-3 \frac{\left(\partial^{3} \rho\right)^{2}}{\left(\partial^{2} \rho\right)^{4}}\right) \partial \psi^{+} \psi^{+}-\frac{8}{3} \frac{\partial^{3} \psi^{+} \psi^{+}}{\left(\partial^{2} \rho\right)^{2}}\right. \\
& \left.\quad+\frac{4 \partial^{3} \rho}{\left(\partial^{2} \rho\right)^{3}} \partial^{2} \psi^{+} \psi^{+}+\frac{4}{3} \frac{\partial^{3} \psi^{+} \partial^{2} \psi^{+} \partial \psi^{+} \psi^{+}}{\left(\partial^{2} \rho\right)^{4}}\right\}\left(z_{I}\right) \\
& + \text { c.c. }] .
\end{aligned}
$$

The path integral over $\psi^{ \pm}, \bar{\psi}^{ \pm}$can be computed by treating $S_{\text {int }}$ perturbatively. Since $S_{\text {int }}$ involves only $\psi^{+}$, the perturbation series terminates at a finite order. 


\section{C.2 A proof of equality of (3.14) and (3.15)}

In this appendix, we show that (3.14) is equal to (3.15). In the case $Q=0$, this equality implies that (3.2) is equal to (3.7). Proving this can be done by using a fermionic charge ${ }^{5}$

$$
\hat{Q}^{\prime} \equiv \oint \frac{d z}{2 \pi i}\left[-\frac{b}{4 \partial \rho}\left(i X_{\mathrm{L}}^{+}-\frac{1}{2} \rho\right)(z)+\frac{\beta}{2 \partial \rho} \psi^{+}(z)\right],
$$

and its antiholomorphic counterpart $\hat{\bar{Q}}^{\prime}$. Here $\left(i X_{\mathrm{L}}^{+}-\frac{1}{2} \rho\right)(z)$ is defined as

$$
\left(i X_{\mathrm{L}}^{+}-\frac{1}{2} \rho\right)(z) \equiv \int_{w_{0}}^{z} d z^{\prime}\left(i \partial X^{+}-\frac{1}{2} \partial \rho\right)\left(z^{\prime}\right)
$$

with a generic point $w_{0}$ on the surface. $\left(i X_{\mathrm{L}}^{+}-\frac{1}{2} \rho\right)(z)$ thus defined is single valued on the surface in the correlation functions we consider here because $-\frac{i}{2} \rho$ coincides with the expectation value of $X_{\mathrm{L}}^{+}$in the presence of the sources $e^{-i p_{r}^{+} X^{-}}$. In order to use $\hat{Q}^{\prime}$, we need to rewrite the ghost part of the correlation function. Inserting

$$
1=\left|\oint_{w_{0}} \frac{d z}{2 \pi i} \frac{b}{\partial \rho}(z) \partial \rho c\left(w_{0}\right)\right|^{2}
$$

into (3.2) and deforming the contours of the antighost insertions, (3.2) is transformed into

$$
\begin{aligned}
& \int[\left.d X^{\mu} d \psi^{\mu} d \bar{\psi}^{\mu} d b d \bar{b} d c d \bar{c} d \beta d \bar{\beta} d \gamma d \bar{\gamma}\right]_{g_{z \bar{z}}^{\mathrm{A}}} e^{-S^{\mathrm{tot}}} \\
& \times \partial \rho c\left(w_{0}\right) \bar{\partial} \bar{\rho} \bar{c}\left(\bar{w}_{0}\right) \\
& \quad \prod_{j=1}^{g}\left[\left(\oint_{\alpha_{j}} \frac{d z}{\partial \rho} b_{z z}+\oint_{\alpha_{j}} \frac{d \bar{z}}{\bar{\partial} \bar{\rho}} b_{\bar{z} \bar{z}}\right)\left(\oint_{\beta_{j}} \frac{d z}{\partial \rho} b_{z z}+\oint_{\beta_{j}} \frac{d \bar{z}}{\bar{\partial} \bar{\rho}} b_{\bar{z} \bar{z}}\right)\right] \\
& \quad \times \prod_{I}\left[\oint_{z_{I}} \frac{d z}{2 \pi i} \frac{b}{\partial \rho}(z) X\left(z_{I}\right) \oint_{\bar{z}_{I}} \frac{d \bar{z}}{2 \pi i} \frac{\bar{b}}{\partial \bar{\rho}}(\bar{z}) \bar{X}\left(\bar{z}_{I}\right)\right] \prod_{r} e^{-\frac{i Q^{2}}{\alpha_{r}} \mathcal{X}^{+}}\left(\hat{\tilde{\mathbf{z}}}_{I^{(r)}}, \hat{\overline{\mathbf{z}}}_{I^{(r)}}\right) \\
& \quad \times V_{1}^{(-2,-1)}\left(Z_{1}, \bar{Z}_{1}\right) V_{2}^{(0,-1)}\left(Z_{2}, \bar{Z}_{2}\right) \prod_{r=3}^{N}\left[V_{r}^{(-1,-1)}\left(Z_{r}, \bar{Z}_{r}\right)\right] .
\end{aligned}
$$

Here $\alpha_{j}$ and $\beta_{j}$ are chosen so that they form a canonical basis of the first homology group of the Riemann surface. Using $\hat{Q}^{\prime}$, the operators inserted at $z=z_{I}$ can be expressed as

$$
\begin{aligned}
\oint_{z_{I}} & \frac{d z}{2 \pi i} \frac{b}{\partial \rho}(z) X\left(z_{I}\right) \prod_{r}^{\prime} e^{-\frac{i Q^{2}}{\alpha_{r}} \mathcal{X}^{+}}\left(\hat{\tilde{\mathbf{z}}}_{I^{(r)}}, \hat{\tilde{\mathbf{z}}}_{I^{(r)}}\right) \\
= & -\oint_{z_{I}} \frac{d z}{2 \pi i} \frac{b}{\partial \rho}(z) e^{\phi} T_{\mathrm{F}}^{\mathrm{LC}}\left(z_{I}\right) \prod_{r}^{\prime} e^{-\frac{i Q^{2}}{\alpha_{r}} \mathcal{X}^{+}}\left(\hat{\tilde{\mathbf{z}}}_{I^{(r)}}, \hat{\tilde{\mathbf{z}}}_{I^{(r)}}\right) \\
& -\left\{\hat{Q}^{\prime}, \oint_{z_{I}} \frac{d z}{2 \pi i} \frac{b}{\partial \rho}(z) \oint_{z_{I}} \frac{d w}{2 \pi i} \frac{A(w)}{w-z_{I}} e^{\phi}\left(z_{I}\right) \prod_{r}^{\prime} e^{-\frac{i Q^{2}}{\alpha_{r}} \mathcal{X}^{+}}\left(\hat{\tilde{\mathbf{z}}}_{I^{(r)}}, \hat{\tilde{\mathbf{z}}}_{I^{(r)}}\right)\right\} \\
& +\frac{1}{4} \oint_{z_{I}} \frac{d z}{2 \pi i} \frac{b}{\partial \rho}(z) \oint_{z_{I}} \frac{d w}{2 \pi i} \frac{\partial \rho \psi^{-}(w)}{w-z_{I}} e^{\phi}\left(z_{I}\right) \prod_{r}^{\prime} e^{-\frac{i Q^{2}}{\alpha_{r}} \mathcal{X}^{+}}\left(\hat{\tilde{\mathbf{z}}}_{I^{(r)}}, \hat{\tilde{\mathbf{z}}}_{I^{(r)}}\right)
\end{aligned}
$$

\footnotetext{
${ }^{5}$ This fermionic charge was used in [16].
} 
Here the prime in $\prod^{\prime}$ means that the product is taken over those $r$ which satisfy

$$
z_{I^{(r)}}=z_{I}
$$

and

$$
\begin{gathered}
A(w)=-i \partial X^{+} \partial \rho \gamma(w)-2 \partial(\partial \rho c) \psi^{-}(w) \\
-\frac{d-10}{4} i\left[\left(\frac{5\left(\partial^{2} X^{+}\right)^{2}}{4\left(\partial X^{+}\right)^{3}}-\frac{\partial^{3} X^{+}}{2\left(\partial X^{;}\right)^{2}}\right)(-2 \partial \rho \gamma)-\frac{2 \partial^{2} X^{+}}{\left(\partial X^{+}\right)^{2}} \partial(-2 \partial \rho \gamma)\right. \\
\left.+\frac{\partial^{2}(-2 \partial \rho \gamma)}{\partial X^{+}}-\frac{(-2 \partial \rho \gamma) \partial \psi^{+} \partial^{2} \psi^{+}}{2\left(\partial X^{+}\right)^{3}}\right](w) .
\end{gathered}
$$

Substituting (C.19) into (C.18) and using the commutators

$$
\begin{aligned}
& \left\{\hat{Q}^{\prime}, \hat{Q}^{\prime}\right\}=0, \\
& \left\{\hat{Q}^{\prime}, c\left(w_{0}\right)\right\}=0, \\
& \left\{\hat{Q}^{\prime}, b(z)\right\}=0 \\
& {\left[\hat{Q}^{\prime}, V_{r}^{(p,-1)}\right]=0 \quad(p=-2,-1,0),} \\
& \left\{\hat{Q}^{\prime}, \oint_{z_{I}} \frac{d z}{2 \pi i} \frac{b}{\partial \rho}(z) e^{\phi} T_{\mathrm{F}}^{\mathrm{LC}}\left(z_{I}\right) \prod_{r}^{\prime} e^{-\frac{i Q^{2}}{\alpha_{r}} \mathcal{X}^{+}}\left(\hat{\tilde{\mathbf{z}}}_{I^{(r)}}, \hat{\tilde{\mathbf{z}}}_{I^{(r)}}\right)\right\}=0, \\
& \left\{\hat{Q}^{\prime}, \oint_{z_{I}} \frac{d z}{2 \pi i} \frac{b}{\partial \rho}(z) \oint_{z_{I}} \frac{d w}{2 \pi i} \frac{\partial \rho \psi^{-}(w)}{w-z_{I}} e^{\phi}\left(z_{I}\right) \prod_{r}^{\prime} e^{-\frac{i Q^{2}}{\alpha_{r}} \mathcal{X}^{+}}\left(\hat{\tilde{\mathbf{z}}}_{I^{(r)}}, \hat{\tilde{\mathbf{z}}}_{I^{(r)}}\right)\right\}=0,
\end{aligned}
$$

we can show that (C.18) is equal to

$$
\begin{aligned}
\int[ & \left.d X^{\mu} d \psi^{\mu} d \bar{\psi}^{\mu} d b d \bar{b} d c d \bar{c} d \beta d \bar{\beta} d \gamma d \bar{\gamma}\right]_{g_{z \bar{z}}^{\mathrm{A}}} e^{-S^{\mathrm{tot}}} \\
& \times \partial \rho c\left(w_{0}\right) \bar{\partial} \bar{\rho} \bar{c}\left(\bar{w}_{0}\right) \\
& \times \prod_{j=1}^{g}\left[\left(\oint_{\alpha_{j}} \frac{d z}{\partial \rho} b_{z z}+\oint_{\alpha_{j}} \frac{d \bar{z}}{\bar{\partial} \bar{\rho}} b_{\bar{z} \bar{z}}\right)\left(\oint_{\beta_{j}} \frac{d z}{\partial \rho} b_{z z}+\oint_{\beta_{j}} \frac{d \bar{z}}{\bar{\partial} \bar{\rho}} b_{\bar{z} \bar{z}}\right)\right] \\
& \times \prod_{I} \oint_{z_{I}} \frac{d z}{2 \pi i} \frac{b}{\partial \rho}(z)\left[-e^{\phi} T_{\mathrm{F}}^{\mathrm{LC}}\left(z_{I}\right)+\frac{1}{4} \oint_{z_{I}} \frac{d w}{2 \pi i} \frac{\partial \rho \psi^{-}(w)}{w-z_{I}} e^{\phi}\left(z_{I}\right)\right] \\
& \times \prod_{I}\left[\oint_{\bar{z}_{I}} \frac{d \bar{z}}{2 \pi i} \frac{\bar{b}}{\partial \bar{\rho}}(\bar{z}) \bar{X}\left(\bar{z}_{I}\right)\right] \prod_{r} e^{-\frac{i Q^{2}}{\alpha_{r}} \mathcal{X}^{+}}\left(\hat{\tilde{\mathbf{z}}}_{I^{(r)}}, \hat{\tilde{\mathbf{z}}}_{I^{(r)}}\right) \\
& \times V_{1}^{(-2,-1)}\left(Z_{1}, \bar{Z}_{1}\right) V_{2}^{(0,-1)}\left(Z_{2}, \bar{Z}_{2}\right) \prod_{r=3}^{N}\left[V_{r}^{(-1,-1)}\left(Z_{r}, \bar{Z}_{r}\right)\right] .
\end{aligned}
$$

The correlation functions of $\psi^{ \pm}$which appear in (C.23) can be calculated using (C.13) treating $S_{\text {int }}$ perturbatively. Since $\partial \rho\left(z_{I}\right)=0, \oint_{z_{I}} \frac{d w}{2 \pi i} \frac{\partial \rho \psi^{-}(w)}{w-z_{I}}$ vanishes unless $\psi^{-}(w)$ becomes singular at $w=z_{I}$. Hence if the $\psi^{+}\left(Z_{1}\right)$ in $V_{1}^{(-2,-1)}\left(Z_{1}, \bar{Z}_{1}\right)$ is contracted with 
the $\psi^{-}(w)$ in $\oint_{z_{I}} \frac{d w}{2 \pi i} \frac{\partial \rho \psi^{-}(w)}{w-z_{I}}$, the contour integral over $w$ vanishes. Therefore only the terms which involve contraction of the $\psi^{+}\left(Z_{1}\right)$ in $V_{1}^{(-2,-1)}\left(Z_{1}, \bar{Z}_{1}\right)$ with the $\psi^{-}\left(Z_{2}\right)$ in $V_{2}^{(0,-1)}\left(Z_{2}, \bar{Z}_{2}\right)$ survive. The $\psi^{-}(w)$ in $\oint_{z_{I}} \frac{d w}{2 \pi i} \frac{\partial \rho \psi^{-}(w)}{w-z_{I}}$ should be contracted with $\partial^{n} \psi^{+}\left(z_{I}\right)$ involved in $e^{-\frac{i Q^{2}}{\alpha_{r}} \mathcal{X}^{+}}\left(\hat{\tilde{\mathbf{z}}}_{I^{(r)}}, \hat{\tilde{\mathbf{z}}}_{I^{(r)}}\right)$ or $S_{\text {int }}$ but doing so induces another contraction

$$
\oint_{z_{J}} \frac{d w}{2 \pi i} \frac{1}{w-z_{J}} \partial \rho \psi^{-}(w) \partial^{n} \psi^{+}\left(z_{I}\right)
$$

with $z_{J} \neq z_{I}$, because $e^{-\frac{i Q^{2}}{\alpha_{r}} \mathcal{X}^{+}}\left(\hat{\tilde{\mathbf{z}}}_{I^{(r)}}, \hat{\tilde{\mathbf{z}}}_{I^{(r)}}\right)$ and $S_{\text {int }}$ are Grassmann even. Hence we conclude that $\oint_{z_{I}} \frac{d w}{2 \pi i} \frac{\partial \rho \psi^{-}(w)}{w-z_{I}}$ in (C.23) does not contribute to the path integral. We can do the same thing for the antiholomorphic part and prove that the $X\left(z_{I}\right), \bar{X}\left(\bar{z}_{I}\right)$ which appear in (C.18) can be replaced by $-e^{\phi} T_{\mathrm{F}}^{\mathrm{LC}}\left(z_{I}\right),-e^{\bar{\phi}} \bar{T}_{\mathrm{F}}^{\mathrm{LC}}\left(\bar{z}_{I}\right)$ for all $I$. By deforming the contours of the antighost insertions back, we can see that (3.14) is equal to (3.15).

Open Access. This article is distributed under the terms of the Creative Commons Attribution License (CC-BY 4.0), which permits any use, distribution and reproduction in any medium, provided the original author(s) and source are credited.

\section{References}

[1] A. Sen, BV master action for heterotic and type II string field theories, JHEP 02 (2016) 087 [arXiv: 1508.05387] [INSPIRE].

[2] A. Sen, Gauge invariant $1 P I$ effective action for superstring field theory, JHEP 06 (2015) 022 [arXiv: 1411.7478] [INSPIRE].

[3] A. Sen, Gauge invariant 1PI effective superstring field theory: inclusion of the Ramond sector, JHEP 08 (2015) 025 [arXiv: 1501.00988] [INSPIRE].

[4] C. de Lacroix, H. Erbin, S.P. Kashyap, A. Sen and M. Verma, Closed superstring field theory and its applications, Int. J. Mod. Phys. A 32 (2017) 1730021 [arXiv:1703.06410] [InSPIRE].

[5] A. Sen, Background independence of closed superstring field theory, JHEP 02 (2018) 155 [arXiv: 1711.08468] [INSPIRE].

[6] B. Zwiebach, Closed string field theory: quantum action and the B-V master equation, Nucl. Phys. B 390 (1993) 33 [hep-th/9206084] [InSPIRE].

[7] K. Aoki, E. D'Hoker and D.H. Phong, Unitarity of closed superstring perturbation theory, Nucl. Phys. B 342 (1990) 149 [inSPIRE].

[8] J. Greensite and F.R. Klinkhamer, New interactions for superstrings, Nucl. Phys. B 281 (1987) 269 [INSPIRE].

[9] J. Greensite and F.R. Klinkhamer, Contact interactions in closed superstring field theory, Nucl. Phys. B 291 (1987) 557 [INSPIRE].

[10] J. Greensite and F.R. Klinkhamer, Superstring amplitudes and contact interactions, Nucl. Phys. B 304 (1988) 108 [inSPIRE].

[11] M.B. Green and N. Seiberg, Contact interactions in superstring theory, Nucl. Phys. B 299 (1988) 559 [inSPIRE]. 
[12] C. Wendt, Scattering amplitudes and contact interactions in Witten's superstring field theory, Nucl. Phys. B 314 (1989) 209 [InSPIRE].

[13] N. Ishibashi and K. Murakami, Multiloop amplitudes of light-cone gauge NSR string field theory in noncritical dimensions, JHEP 01 (2017) 034 [arXiv:1611.06340] [INSPIRE].

[14] N. Ishibashi, Light-cone gauge superstring field theory in linear dilaton background, PTEP 2017 (2017) 033B01 [arXiv:1605.04666] [INSPIRE].

[15] Y. Baba, N. Ishibashi and K. Murakami, Light-cone gauge superstring field theory and dimensional regularization, JHEP 10 (2009) 035 [arXiv:0906.3577] [INSPIRE].

[16] N. Ishibashi and K. Murakami, Light-cone gauge NSR strings in noncritical dimensions II Ramond sector, JHEP 01 (2011) 008 [arXiv:1011.0112] [INSPIRE].

[17] E. D'Hoker and S.B. Giddings, Unitary of the closed bosonic Polyakov string, Nucl. Phys. B 291 (1987) 90 [INSPIRE].

[18] S.B. Giddings and S.A. Wolpert, A triangulation of moduli space from light cone string theory, Commun. Math. Phys. 109 (1987) 177 [INSPIRE].

[19] E. D'Hoker and D.H. Phong, The geometry of string perturbation theory, Rev. Mod. Phys. 60 (1988) 917 [InSPIRE].

[20] N. Ishibashi and K. Murakami, Multiloop amplitudes of light-cone gauge bosonic string field theory in noncritical dimensions, JHEP 09 (2013) 053 [arXiv:1307.6001] [INSPIRE].

[21] S. Arakelov, Intersection theory of divisors on an arithmetic surface, Math. USSR Izv. 8 (1974) 1167.

[22] E.P. Verlinde and H.L. Verlinde, Multiloop calculations in covariant superstring theory, Phys. Lett. B 192 (1987) 95 [INSPIRE].

[23] A. Sen and E. Witten, Filling the gaps with PCO's, JHEP 09 (2015) 004 [arXiv: 1504.00609] [INSPIRE].

[24] A. Sen, Off-shell amplitudes in superstring theory, Fortsch. Phys. 63 (2015) 149 [arXiv: 1408.0571] [INSPIRE].

[25] E. Witten, More on superstring perturbation theory: an overview of superstring perturbation theory via super Riemann surfaces, arXiv:1304.2832 [INSPIRE].

[26] A. Sen, Supersymmetry restoration in superstring perturbation theory, JHEP 12 (2015) 075 [arXiv: 1508.02481] [INSPIRE].

[27] J.J. Atick and A. Sen, Spin field correlators on an arbitrary genus Riemann surface and nonrenormalization theorems in string theories, Phys. Lett. B 186 (1987) 339 [INSPIRE].

[28] N. Berkovits, Calculation of scattering amplitudes for the Neveu-Schwarz model using supersheet functional integration, Nucl. Phys. B 276 (1986) 650 [InSPIRE].

[29] N. Berkovits, Supersheet functional integration and the interacting Neveu-Schwarz string, Nucl. Phys. B 304 (1988) 537 [inSPIRE].

[30] Y. Baba, N. Ishibashi and K. Murakami, Light-cone gauge superstring field theory and dimensional regularization II, JHEP 08 (2010) 102 [arXiv:0912.4811] [INSPIRE].

[31] E.P. Verlinde and H.L. Verlinde, Chiral bosonization, determinants and the string partition function, Nucl. Phys. B 288 (1987) 357 [INSPIRE].

[32] J.D. Fay, Theta functions on Riemann surfaces, Lect. Notes Math. 352, Springer-Verlag, Berlin Heidelberg Germany, (1973). 